\title{
Crossflow Instability on a Wedge-Cone at Mach 3.5
}

\author{
George B. Beeler ${ }^{1}$, Stephen P. Wilkinson ${ }^{2}$, P. Balakumar ${ }^{3}$ \\ NASA Langley Research Center,Hampton,Virginia,23681 \\ and \\ Keith S. McDaniel, ${ }^{4}$ \\ Analytical Mechanics Associates Inc., Hampton, Virginia, 23681
}

\begin{abstract}
As a follow-on activity to the HyBoLT flight experiment, a six degree half angle wedge-cone model at zero angle of attack has been employed to experimentally and computationally study the boundary layer crossflow instability at Mach 3.5 under low disturbance freestream conditions. Computed meanflow and linear stability analysis results are presented along with corresponding experimental Pitot probe data. Using a model-mounted probe survey apparatus, data acquired to date show a well defined stationary crossflow vortex pattern on the flat wedge surface. This effort paves the way for additional detailed, calibrated flow field measurements of the crossflow instability, both stationary and traveling modes, and transition-to-turbulence under quiet flow conditions as a means of validating existing stability theory and providing a foundation for dynamic flight instrumentation development.
\end{abstract}

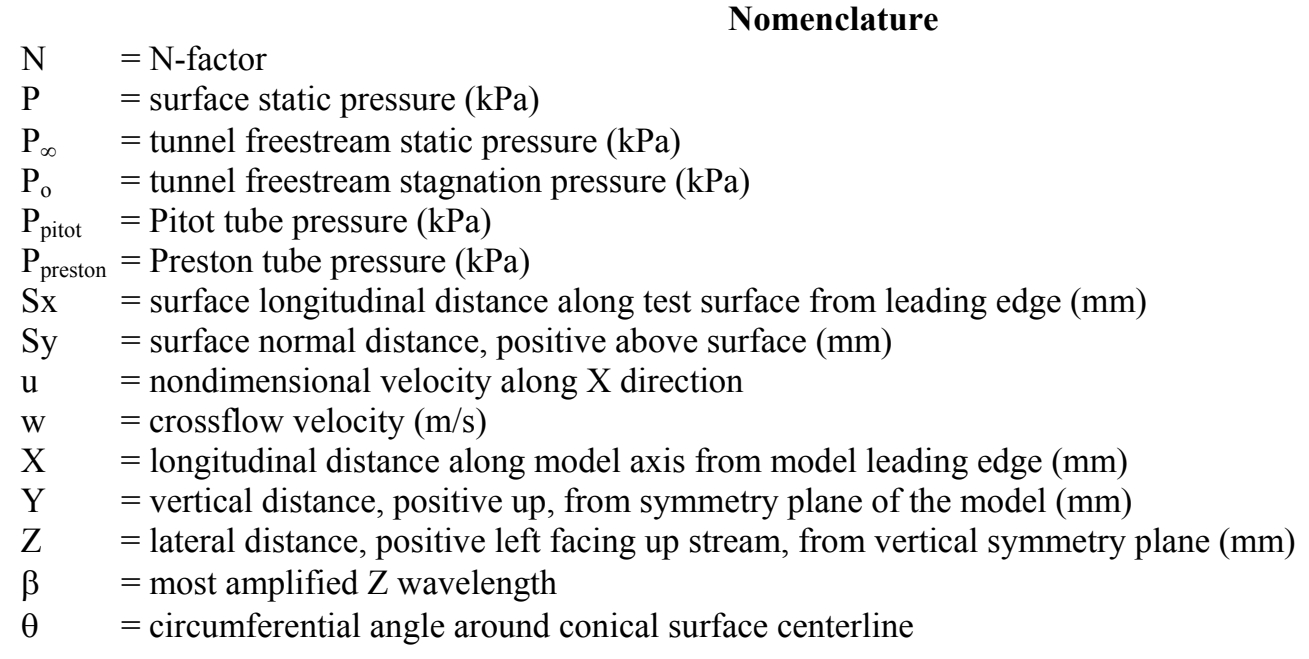

\footnotetext{
${ }^{1}$ Research Scientist, Flow Physics and Control Branch, M/S 170, AIAA Senior Member.

${ }^{2}$ Research Scientist, Flow Physics and Control Branch, M/S 170, AIAA Senior Member.

${ }^{3}$ Research Scientist, Flow Physics and Control Branch, M/S 170, AIAA Senior Member.

${ }^{4}$ Research Scientist, Hypersonic Air Breathing Propulsion Branch, M/S 168, AIAA Senior Member.
} 


\section{Introduction}

$\mathrm{T}$ HE crossflow instability mode is the dominant transition mechanism on swept wings and, therefore, has tremendous technological importance with respect to flight efficiency at both low and high speeds. It is one of four fundamental boundary layer transition eigenmodes predicted by linear stability theory, the others being $1^{\text {st }}, 2^{\text {nd }}$, and Görtler modes, and as such has received significant theoretical and experimental attention. A relatively recent review article on crossflow dominated transition is provided by Saric and Reed. ${ }^{1}$ The crossflow instability stems from a cross-stream pressure gradient and appears as co-rotating vortices within the boundary layer aligned approximately with the external flow streamlines. It is observed in two varieties - stationary and traveling. The stationary mode is extremely sensitive to surface roughness and micrometer-sized roughness elements are sufficient to initiate and anchor such vortices. ${ }^{2,3}$ The traveling mode is initiated by freestream acoustic and vorticity disturbances, which tends to be minimized in low disturbance environments such as flight and low disturbance wind tunnels. ${ }^{4}$

Current research on the crossflow mode is focused in three main areas: flow control (particularly schemes based on excitation of less amplified wave numbers using micrometer-sized roughness arrays that increase the transition Reynolds number); CFD investigations of receptivity, growth, and breakdown; and experiments providing data for code validation, control methodologies, and (in general) fundamental aspects of the crossflow problem. This paper deals with the second and third areas with fundamental numerical and experimental efforts aimed at providing a better understanding of crossflow dominated transition for the supersonic case. In particular, we employ a novel method of creating crossflow on a flat surface using a wedge-cone geometry. This geometry is advantageous in that a robust stationary crossflow pattern has been observed and transition has been verified making this potentially a new benchmark model for supersonic crossflow research.

This investigation originated with the NASA/ATK ALV-X1 sounding rocket flight experiment carrying the Hypersonic Boundary Layer Transition experiment (HyBoLT) and Sub-Orbital Aerodynamic Re-entry Experiments (SOAREX-VI) from NASA Wallops on August 22, 2008, ${ }^{5,6,7}$ The HyBoLT test article was a symmetric wedge flanked by tangent cones with different transition experiments on each face. On one face, termed "Side A", a smooth wall crossflow experiment was designed to detect both stationary and traveling crossflow modes. On the other side termed "Side B", a roughness experiment was installed in support of the Space Shuttle boundary layer transition investigations. Unfortunately, the rocket had to be destroyed 20 seconds into the flight due to guidance issues $^{6}$ and no useful boundary layer transition data were acquired. However, the wind tunnel portion of the "Side A" study continues forming the basis of this report.

To enable detailed measurements of stationary crossflow, the subject model was tested in the NASA Langley Mach 3.5 Supersonic Low Disturbance Tunnel (SLDT) ${ }^{8}$ in the rapid expansion 2D nozzle shown in Fig. 1. The quiet freestream of the SLDT with pressure fluctuations less than $0.1 \%$, potentially limits the formation of traveling crossflow modes thereby facilitating detailed study of the flight-relevant stationary mode. High quality boundary layer profiles were acquired during the first entry, which show the formation of a repeatable, stationary mode crossflow vortex pattern. This first entry showed evidence of full transition near the trailing edge of the model using a fixed surface Preston tube. A second entry with modifications such that boundary layer surveys could be obtained further downstream from the first entry has shown the path of the vortices propagating aft and the repeatability of the disturbance flow over this model.

\section{Experimental Setup}

The model geometry is a six degree half-angle, symmetric wedge flanked by spanwise-tangent, inclined and sheared cones (the cross sections are circular in planes perpendicular to the model symmetry axis) with dimensions as shown in Fig. 2. This is an approximate $27 \%$ scale model of the forward half of the HyBoLT test article. The model length was limited to $254 \mathrm{~mm}$ (10 in.) to be able to start the facility and to avoid shock wave reflections on the model test region. A picture of the model as tested without the extension shelf is shown in Fig. 3.

The model has a sharp leading edge with a radius of $50 \mu \mathrm{m}(0.002 \mathrm{in}$.). Surface finish was specified as $0.1 \mu \mathrm{m}(4$ x $10^{-6}$ in.) rms and local meaurements of surface varied from $0.025-0.075 \mu \mathrm{m}\left(1-3 \times 10^{-6}\right.$ in.) with waviness less than $12.7 \mu \mathrm{m}$ per $25.4 \mathrm{~mm}$ (0.0005 in. per in.) length. The model was fabricated from solid stainless steel with a large instrumentation access cavity leaving a solid leading edge length of $43 \mathrm{~mm}$. The interior cavity width is limited by the solid conical sides with an allowance for the $2.54 \mathrm{~mm}(0.1 \mathrm{in}$.) thick lower surface hatch. The base of the model is $26 \mathrm{~mm}$ thick where the sting attaches. The leading edge corners were specified to be sharp. However, the corners were inadvertently rounded to $2 \mathrm{~mm}(0.08 \mathrm{in}$.) on the starboard side and $2.5 \mathrm{~mm}(0.1 \mathrm{in}$.) on the port side in 
planform radius during construction. It was decided to leave the radii after computations described in the meanflow calculation section showed that a sharp or $2.5 \mathrm{~mm}$ radius corner generate almost identical surface pressure distributions.

The model is instrumented with 61 static pressure taps and 13 thermocouples. Thirty of the pressure taps are distributed along the centerline of the two (upper and lower) flat wedge surfaces at identical Sx stations (from 46.76 $\mathrm{mm}$ to $224.56 \mathrm{~mm}$ in $12.7 \mathrm{~mm}$ increments from the leading edge) and were used to zero the angle of attack. The remaining pressure taps are in three spanwise rows and are continued around the starboard (right looking upstream) conical side of the model at $22.5^{\circ}$ intervals to $112.5^{\circ}$ from the flat test surface around the cone centerline. The spanwise taps are in planes normal to the primary test surface and are in the test region in only one quadrant of the model. This leaves an unbroken surface in the other three quadrants of the model for boundary layer studies clear of surface disturbances. The taps are located as shown in Fig. 2 and in detail in Table 1.

Two $34.47 \mathrm{kPa}(5 \mathrm{psi})$ differential pressure sensor modules were used to measure the surface pressures. The centerline taps used for angle of attack assessment were all on a single Digital Temperature Compensation (DTC) module and the remainder of the spanwise taps were measured with a standard module. These modules were calibrated over a $\pm 6.895 \mathrm{kPa}( \pm 1 \mathrm{psi})$ differential pressure range and all readings reported were within this range throughout testing. The differential pressure modules were referenced to the box pressure surrounding the open jet nozzle measured using a 100 Torr absolute sensor. The DTC has an accuracy specification of $\pm 0.03 \%$ of full scale and the standard module is $\pm 0.05 \%$ of full scale while the 100 Torr sensor has a tolerance of $\pm 0.05 \%$ of reading. Since the peak surface pressure expected is $2.18 \%$ of the stagnation pressure and the maximum stagnation pressure is $689 \mathrm{kPa}(100 \mathrm{psia})$, the accuracy of the measurements should be better than $\pm 18 \mathrm{~Pa}$ on the centerline measurements and $\pm 25 \mathrm{~Pa}$ on the spanwise ports, assuming the errors are additive.

The model is also instrumented internally with 13 type $\mathrm{T}$ thermocouples for measuring bulk model temperature to verify model equilibrium temperatures. During testing, the thermocouples were allowed to stabilize before data were acquired. Three of these thermocouples are embedded in the solid leading edge, two in the solid conical side opposite from the pressure taps and four each in a rectangular pattern distributed in two rows $(57.15$ and $120.65 \mathrm{~mm}$ from the leading edge) at $41.275 \mathrm{~mm}$ on either side of the test surface centerline on both the upper and lower test surface of the model. Table 2 summarizes the locations of the thermocouples.

The model is fitted with a sting-mounted three-axis traverse system for surveying the flat model surfaces. The traverse is similar to one presented by Owens, Kegerise, and Wilkinson ${ }^{9}$ for a cone and is almost identical to that used on a flat plate by Kegerise, Owens and King. ${ }^{10}$ A picture of the model with the integral traverse is shown in Fig. 3 (without the extension shelf shown in Fig. 2). The traverse is aligned with the upper flow surface of the model and during the first tunnel entry could translate probes from forward of the leading edge to $168.53 \mathrm{~mm}(6.635 \mathrm{in}$.) downstream of the leading edge of the model. Positions further aft were not possible with the initial configuration of the model as the traverse relies on a solid Ultra High Molecular Weight Plastic (UHMWP) pin to support the pivoting traverse head and the pin would be off the model if the traverse was moved further aft. The support pin remains in contact with the model surface to maintain a stable standoff distance of the probe arm during measurements. In the second entry with the extension shelf, the full test region shown in Fig. 2 could be scanned. Calibration of the pivoting head at the end of the probe arm was performed as discussed in Refs. 9 and 10 prior to each entry with resulting resolution of $1.26 \mu \mathrm{m} / \mathrm{step}$ and accuracy of $\pm 5 \mu \mathrm{m}$ using a touch circuit and the Differential Variable Reluctance Transducer described in Ref. 9. Both lateral and wall-normal surveys of the boundary layer in the survey region (for the initial entry) were performed in this effort.

Lateral motion of the traverse was evaluated pre-test. The measured resolution was $0.04 \mathrm{~mm} / \mathrm{step}$ with repeatability of \pm 2 steps once backlash was addressed by unidirectional motion. A significant portion of the first entry was spent getting repeatability of the traverse lateral scans under load through adjustment of the support pin. Lateral traverse alignment ( $\mathrm{Z}$ direction) was an issue during the first entry. We discovered that the higher operational pressures could move the traverse rapidly during tunnel shutdown and these motions were not accurately detected by the incremental encoders. This, in conjunction with a reference stop that was also shifted by this load resulted in position offsets outboard up to approximately $1 \mathrm{~mm}$. This uncertainty was corrected on the second entry with an unconditionally stable reference stop. Typical uncertainty in lateral motion from point to point is significantly more accurate at approximately $80 \mu \mathrm{m}$.

In the second entry with this model, we incorporated an extension shelf for the upper test surface of the model. The extension shelf, as shown in Fig. 2, allows the probe to be positioned near the downstream end of the model over the full test region shown. Figure 4 shows a picture with this extension shelf showing the downstream end of 
the model and the traverse support pin properly supporting the traverse far aft of the first entry. The shelf is aligned with the existing test surface within $12.7 \mu \mathrm{m}$, which is slightly larger than the $10 \mu \mathrm{m}$ (Sy) traverse repeatability.

In both entries, the model was placed near the leading edge of the uniform flow of the nozzle and aligned to within $0.127 \mathrm{~mm}(0.005 \mathrm{in}$.) of the nozzle symmetry plane and $8.7 \mathrm{~mm}(0.343 \mathrm{in}$.) downstream of the uniform core origin in the nozzle. This placement assures the model leading edge was in uniform quiet flow and places the trailing edge of the model approximately even with the nozzle exit. Although this does not allow for Schlieren images over the model surface, it provides the longest extent of quiet flow possible over the model providing this effort with the best chance of capturing stationary modes.

Surveys were performed using a flattened Pitot probe with an external height of $0.1 \mathrm{~mm}$ and width of $0.3 \mathrm{~mm}$ connected to a $172 \mathrm{kPa}(25 \mathrm{psi})$ miniature absolute pressure sensor monitored throughout the test for drift. An electrical touch circuit was employed where the Pitot probe lightly touches (i.e., "fouls") on the metal surface of the model, establishing the initial wall-normal position. This was done for each wall-normal survey resulting in typical initial position repeatability of better than $10 \mu \mathrm{m}$. During lateral scans, the probe height above the wall was set using the fouling technique at the inboard station. This height would often vary over the length of the scan, primarily due to the traverse support pin not remaining at the same angle throughout the scan as it bears a significant load. The flattened Pitot probe and pressure sensor are the same hardware as was used in the previously referenced experiments by Owens et al. ${ }^{9}$ and Kegerise et al. ${ }^{10}$ for boundary layer and meanflow measurements. The focus of their work on the Pitot was to develop a probe that would cause minimum distortion to the boundary layer.

Throughout this test, data were acquired at stagnation pressures from approximately 172 to $689 \mathrm{kPa}$ (25 to 100

psi). This is a significant increase in pressure load over the previous efforts ${ }^{9,10}$ with this traverse design. Vibration of the probe ${ }^{9,10}$ became too large at the higher stagnation pressures in this test to use hot-wires. Noticeable wear was found on the fouling side of the flattened Pitot probe after the test. Thus indicating that lateral vibration levels with this probe traverse design will need to be addressed before hot wire measurements are feasible at the pressure levels required to reach transition onset with this model.

The wedge-cone model was designed to be tested at stagnation pressures up to $689 \mathrm{kPa}$ (100 psi). However, the bulk of the stationary crossflow measurements were performed at $586 \mathrm{kPa}(85 \mathrm{psi})$ to avoid overloading the Pitot pressure sensor in the freestream when performing wall-normal surveys. All testing was performed at a stagnation temperature of $300 \mathrm{~K}$ to coincide with the computations. Typical variations in stagnation test conditions, once stabilized, were within $\pm 0.56{ }^{\circ} \mathrm{C}$ and $\pm 3.5 \mathrm{kPa}$.

In the first entry, lateral Pitot pressure scans were initiated at a height of Sy $=0.4 \mathrm{~mm}$ at the farthest aft position, $\mathrm{Sx}=168.53 \mathrm{~mm}$ (6.635 in.). This height was selected to correspond to the peak in the crossflow eigenfunction. Profiles of the boundary layer were acquired at various longitudinal stations, but were limited in the initial effort to stations up to and including $168.53 \mathrm{~mm}$ (6.635 in.) from the leading edge due to the traverse support pin reaching the aft end of the model.

To determine if transition occurred anywhere on the model during the first entry, a fixed surface Preston tube of diameter $0.22 \mathrm{~mm}$ ( Fig. 5) mounted $243 \mathrm{~mm}$ (9.567 in.) from the leading edge and $57.8 \mathrm{~mm}$ (2.275 in.) from model centerline was used to obtain one set of data. The pressure was measured using the same $172 \mathrm{kPa}(25 \mathrm{psi})$ sensor used for the traversing Pitot measurements. During this run, the tunnel pressure was increased slowly to $689 \mathrm{kPa}$ (100 psi) and paused at intervals to acquire several points before proceeding to the next pressure.

\section{Results and Discussion}

\section{A. Meanflow Calculation}

The meanflow was computed by solving the three-dimensional Navier-Stokes equations using the VULCAN ${ }^{11}$ code. Computations were performed at freestream stagnation pressures of 345,586 , and $689 \mathrm{kPa}(50,85$, and 100 psi) at a freestream stagnation temperature of $300 \mathrm{~K}\left(80^{\circ} \mathrm{F}\right)$ and a Mach number of 3.52. These conditions yield unit Reynolds numbers of $19.7 \times 10^{6} \mathrm{~m}^{-1}, 33.5 \times 10^{6} \mathrm{~m}^{-1}$, and $39.4 \times 10^{6} \mathrm{~m}^{-1}\left(6.0 \times 10^{6} \mathrm{ft}^{-1}, 10.2 \times 10^{6} \mathrm{ft}^{-1}\right.$, and $12.0 \times 10^{6}$ $\left.\mathrm{ft}^{-1}\right)$. All computations were performed with adiabatic wall conditions. The maximum and minimum pressure cases were computed first at both zero and two degrees angle of attack and with sharp (configuration A) and rounded 2.5 $\mathrm{mm}(0.1 \mathrm{in}$.) radius (configuration B) corners as shown in Fig. 6 . The $586 \mathrm{kPa}(85 \mathrm{psi})$ case was computed only with the rounded (configuration B) corner and at zero angle of attack. In this effort, the computational grid was refined to resolve the boundary layer sufficiently for boundary layer stability analysis. Figure 7 shows the refined grid used for the zero angle of attack calculations with every 3rd grid line visible. The solution at $586 \mathrm{kPa}$ and zero angle of attack is shown in Fig. 8 as a function of $\mathrm{X}$ and $\mathrm{Z}$. Note that the model at $254 \mathrm{~mm}$ long is shorter than the computation. The 
corresponding computed bow shock for this solution is shown in Fig. 9 in an isometric view. As expected, a nearly uniform triangular region exists starting from near the leading edge of the model, (essentially the two-dimensional supersonic flow over a six degree wedge) with a short recovery region from the stagnation point. Outside of this 2D region, expansions originating from each corner of the model's leading edge introduce the pressure gradient in the spanwise direction that produces the crossflow inside the boundary layer. Figure 10 depicts the computed crossflow boundary layer profiles at longitudinal station $X=199 \mathrm{~mm}$ and spanwise locations $Z=7.2,15,23,47$, and $69 \mathrm{~mm}$. As expected, the crossflow gradually increases outboard from the centerline of the model and the crossflow profile thicknesses shown are approximately $1 \mathrm{~mm}$ at all the spanwise locations.

\section{B. Linear Stability and Transition Onset}

After the meanflow was computed, linear stability and $\mathrm{N}$-factor calculations were performed using the eMalik3 $\mathrm{d}^{12}$ code. Figure 11 shows the predicted transition fronts that would be caused by the traveling and stationary crossflow instability waves. The $\mathrm{N}$-factors are computed by following group velocity directions as shown by the blue and magenta lines that terminate between $\mathrm{N}=11$ and 12 . The model outline is indicated by the red lines and the flat to conical transition is indicated in red as well. The stationary mode fronts are shown for different Nfactors and are indicated by the dashed black lines at $\mathrm{N}$-factors of $6,6.5,7$, and 8 . Note that the maximum amplifications are outboard, and that disturbances from the centerline do not reach the highest $\mathrm{N}$-factors in the test model length. The computed eigenfunctions for the most amplified stationary mode disturbances are shown in Fig. 12 at $X=198 \mathrm{~mm}$ (7.8 in.) and selected spanwise stations $Z=65,50,35$, and $20 \mathrm{~mm}(2.56,1.97,1.38$, and 0.787 in.). Note that the most amplified wavelengths (denoted by $\beta$ in Fig. 12) increase from $2.2 \mathrm{~mm}$ to $3.0 \mathrm{~mm}$ inboard and that the peak in the curve is invariant near $0.4 \mathrm{~mm}$ until the most outboard station.

\section{Experimental Data - Meanflow}

Surface pressures were measured at a stagnation pressure of $172.4 \mathrm{kPa}(25 \mathrm{psi})$ and the angle of attack was adjusted to minimize the pressure differential from the centerline ports with a $0.635 \mathrm{~mm}(0.025 \mathrm{in}$.) shim resulting in a nose up $0.2^{\circ}$ change. The resulting centerline pressure distributions from the first entry are shown with the computed distributions in Fig. 13. Deviations of the surface pressure from the computation are evident in this plot. After adjustment, the mean of the deviations from CFD is less than $1 \%$ with a maximum of $2.8 \%$. Figure 13 shows that these small angle of attack adjustments do not significantly alter the shape of the centerline pressure distribution, but primarily shift the level of the curves. Notice that after adjustment the upper and lower surface pressures match well from the $\mathrm{Sx}=134 \mathrm{~mm}$ tap downstream and even cross at several locations. Once at this condition, we decided that no further adjustment was necessary. The reasons for the deviations are unclear at this time, but the deviations shown after approximately $0.15 \mathrm{~m}$ were found to move forward when the model was moved aft leading to associating these disturbances primarily with effects originating from the tunnel nozzle flow. It is estimated that the actual angle of attack of the model is within $0.2^{\circ}$ of zero based on this data. All data from this test were acquired with this angle adjustment and appear to repeat with model reinstallation.

The comparison of the computed and measured spanwise pressure distributions at $\mathrm{Sx}=72.16,135.66$ and 199.16 $\mathrm{mm}(2.841,5.341$, and 7.841 in.) are shown in Fig.14. The solid lines are the CFD solutions scaled by freestream pressure that are nearly invariant with stagnation pressure. The solid symbols show the similarly scaled surface pressures measured after the angle of attack adjustment. Note that the majority of the ports particularly in the region from $Z=30$ to $70 \mathrm{~mm}$ are very close to the CFD curves and have a very similar spatial variation. This region of the model contains the region of measurable stationary disturbances found in this investigation. Referring to Table 1 the flat region ends at $76.2 \mathrm{~mm}$ laterally for the first row, $70.587 \mathrm{~mm}$ for the second row and $64.999 \mathrm{~mm}$ for the third row of pressure taps. Outboard of these locations, a suction peak is reached that the CFD does not match on the conical surface. With the exception of near the centerline of the model, the inboard locations follow the predicted surface pressures fairly well. Since these are the driving pressure gradients for the crossflow on the model, crossflow on the outboard flat portion of the model should be reasonably predicted.

\section{Experimental Data - Steady Crossflow}

To locate the crossflow disturbances, a Pitot probe was scanned in the $\mathrm{Z}$ direction at a constant height of $\mathrm{Sy}=0.4$ $\mathrm{mm}$, which was selected from the eigenfunctions of the crossflow as near the maximum amplitude level as seen in Fig. 12. With this lateral data in hand, $\mathrm{Z}$ locations were selected for wall normal profiles to capture the crossflow vortex structures. 
Fig. 15 shows the results of a lateral survey from the first entry through the boundary layer at $\mathrm{Sx}=168.53 \mathrm{~mm}$ and height $\mathrm{Sy}=0.4 \mathrm{~mm}$ (the furthest aft station that could be scanned during the first entry). A stationary disturbance is seen that increases in amplitude in the outboard direction consistent with the higher N-factor outboard as seen in Fig. 11. The lateral survey data from run 86, the red line in this figure, were taken during one continuous tunnel run and all locations recorded are shown by symbols. Points were selected from this survey to perform wall normal surveys, as depicted by the black symbols, in Fig. 15 to capture details of the stationary vortices detected by the lateral survey data. Two separate tunnel runs, 87 and 88, captured eight wall normal surveys at these points shown in Fig. 16. In Fig. 15 the actual values at $S y=0.4 \mathrm{~mm}$ from the wall normal surveys of Fig. 16 are plotted for comparison. Note that the survey data clearly show a similar pattern as was detected during the lateral survey of run 86, demonstrating the repeatable nature of the disturbance. The profiles shown in Fig. 16 (at the target points in the lateral survey) exhibit inflections as would be expected when traversing though a stationary longitudinal vortex in a boundary layer. Using this survey data, we constructed a contour plot of the Pitot data shown in Fig. 17. In this plot, the data points acquired in the surveys are shown by the black mesh lines. Due to the length of the tunnel runtime, the final two surveys in these two runs were cut short in $S y$ at $Z=61$ and $61.5 \mathrm{~mm}$. This plot depicts the clear vortex pattern that has been established on the model, similar to the data captured in subsonic crossflow experiments such as those of Deyhle and Bippes ${ }^{4}$ and Dagenhart and Saric. ${ }^{13}$ Note that this data is captured using a Pitot probe of rather large dimension ( $0.3 \mathrm{~mm}$ lateral width) compared to the apparent size of the vortices from this plot.

In Fig. 18, a lateral scan at $\mathrm{Sx}=168.53 \mathrm{~mm}$ (6.635 in.) from run 20 of the second entry is compared to run 86 from the first entry. Earlier we discussed the uncertainty in $\mathrm{Z}$ location that occurred during the first entry. In the figure, we show the first entry shifted $0.5 \mathrm{~mm}$ for comparison with the later entry. Since the shifted pattern matches the previous entry well, it shows that the model roughness exciting the stationary crossflow disturbance is essentially unchanged from the previous test entry which occurred a year earlier. To wit, the flow over this model can be characterized and used in various tests in the facility with a reasonable expectation of repeatability.

With the extension shelf, lateral scans downstream of the data shown in Fig. 18 were acquired for $\mathrm{Sx}=178.5$ $\mathrm{mm}, 188.5 \mathrm{~mm}, 198.5 \mathrm{~mm}, 211.0 \mathrm{~mm}$, and $223.5 \mathrm{~mm}$ (7.027, 7.421, 7.815, 8.307, and $8.8 \mathrm{in}$.). These scans were acquired at $S y=0.4 \mathrm{~mm}$ from $\mathrm{Z}=50.8$ to $59.7 \mathrm{~mm}$ ( 2 to $2.35 \mathrm{in}$.) limited by the lateral extent of the extension shelf. The results are plotted in Fig. 19 along with the lateral scan data from Fig. 18 (run 20). The curves labeled v1 to v6 in Fig. 19 trace the trajectories of the peak pressure at each longitudinal station until the peaks become ambiguous. As expected, the trajectories migrate outboard going downstream and the wavelengths between the vortices at $\mathrm{Sx}=$ $198.5 \mathrm{~mm}$ vary from approximately $2 \mathrm{~mm}$ to $1.5 \mathrm{~mm}$ from $50.8 \mathrm{~mm}$ outboard. These wavelengths are in the range of the most amplified disturbances of $2.2 \mathrm{~mm}$.

The fixed Preston tube at $Z=57.8 \mathrm{~mm}$ (2.275 in.) near the aft end of the model at $\mathrm{Sx}=243 \mathrm{~mm}(9.57 \mathrm{in}$.) was used to collect the data shown in Fig. 20. Figure 20 clearly indicates that transition occurred before the trailing edge of the model with onset at approximately $430 \mathrm{kPa}(62 \mathrm{psi})$ stagnation pressure at this location. Figure 20 clearly shows all the typical phases of the transition process seen from a Preston tube experiencing transition in the pressure range swept (onset, linear/non-linear growth and breakdown). The method used for determining the approximate transition onset location is shown in Fig. 20.

With this clear indication of transition, the traversing Pitot tube was used to obtain transition onset locations upstream of this point within the scan region. The locations chosen were, $S x=223.5 \mathrm{~mm}(8.80 \mathrm{in}$.) at $Z=55.9 \mathrm{~mm}$ (2.20 in.), $S x=212.7 \mathrm{~mm}$ (8.37 in.) at $Z=57.8 \mathrm{~mm}$ (2.275 in.), and $\mathrm{Sx}=201.9 \mathrm{~mm}$ (7.95 in.) at $Z=59.7 \mathrm{~mm}$ ( $2.350 \mathrm{in}$.), which lie on the stationary mode $\mathrm{N}$-factor $=6$ curve at $586 \mathrm{kPa}(85 \mathrm{psi})$ and are shown in Fig. 11 as red circles. To avoid deforming the flattened Pitot tube, the probe was brought to the wall and fouled after each pressure was reached. Fouling positions the Pitot to within $10 \mu \mathrm{m}$ of the wall with a repeatability of about $\pm 5 \mu \mathrm{m}$ for each pressure acquired. Once fouled, several data points were acquired before raising the probe a safe distance from the wall for the next pressure. The curves acquired in this manner are shown in Fig. 21. Note that the pressure levels are very similar to the levels measured in Fig. 20 even though the integration height is half that of the fixed Preston tube. This fact further confirms the pressure changes shown are due to transition. Although not as clear as the fixed surface Preston tube, using the method shown in Fig. 20 the transition onset is clear for the two aft stations at approximately $\mathrm{P}_{\mathrm{o}}=585 \mathrm{kPa}$ as predicted for $\mathrm{N}$-factor 6 . The furthest forward station shows an early pressure rise at approximately $\mathrm{P}_{\mathrm{o}}=550 \mathrm{kPa}$ and a later rise near $\mathrm{P}_{\mathrm{o}}=580 \mathrm{kPa}$. This data, combined with the boundary layer surveys showing a vortex pattern, suggests that the stationary crossflow instability mode may be the dominant mechanism for transition on this model. 


\section{Summary}

Crossflow transition was studied both experimentally and computationally on a $27 \%$ scale model of the HyBoLT flight test article shortened to $0.254 \mathrm{~m}$ long. The experiment was conducted at Mach 3.5 in the NASA Langley Supersonic Low Disturbance Tunnel (SLDT). The SLDT provides a low disturbance freestream environment to simulate flight conditions. The freestream disturbance levels are important in the study of crossflow transition because it has been theoretically predicted that in conventional tunnels the higher freestream noise environment can potentially trigger the travelling mode crossflow instability, while in flight the stationary mode may dominate.

This study has shown the efficacy of using the crossflow generated by a slender wedge-cone model at zero angle of attack to study supersonic crossflow dominated boundary-layer transition. The benefits of the method include a simple, canonical base flow that can be accurately calculated and the absence of curvature on the test surface facilitates difficult boundary layer diagnostics. The laminar boundary layer thickness on the wind tunnel model was very small, typically less than $1 \mathrm{~mm}$, and considerable care was taken to obtain quantitative boundary layer data on the model. The data acquired indicate that the traverse was able to provide data of a very high quality. Using this model allowed a close coordination between CFD simulation and experiment and has enabled us to take an important initial step toward validating stability theory and furthering our understanding of this important boundary layer instability process. Data from the mean boundary layer surveys has demonstrated a stationary crossflow pattern on the model as predicted from the stability computations for a low disturbance environment. Further data, including for example high frequency hot-wire measurements, need to be obtained to detect whether the travelling mode is also present and to confirm that the stationary mode is dominant in low disturbance environments.

\section{References}

${ }^{1}$ Saric, W. S. and Reed, H. L., "Crossflow Instabilities- Theory and Technology," AIAA Paper 2003-0771, 2003.

2 Saric, W. S. and Reed, H. L., "Supersonic Laminar Flow Control on Swept Wings Using Distributed Roughness," AIAA Paper 2002-0147, 2002.

${ }^{3}$ N.V. Semionov, A.D. Kosinov, and V.Ya. Levchenko., "Experimental Study of Turbulence Beginning and Transition Control in a Supersonic Boundary Layer on Swept Wing," R. Govindarajan (ed.), Sixth IUTAM Symposium on Laminar Turbulent Transition, 2006.

${ }^{4}$ Deyhle, H. and Bippes, H., "Disturbance Growth in an Unstable Three-Dimensional Boundary Layer and its Dependence on Initial Conditions," J. Fluid Mech.316:73-113, 1996.

5 Berry, S. A., Kimmel, R., and Reshotko, E., "Recommendations for Hypersonic Boundary Layer Transition Flight Testing,", AIAA Paper 2011-3415, June 2011.

${ }^{6}$ Chen, Fang-Jenq, Berry, S. A., "HyBoLT Flight Experiment", NASA TM-2010-216725, 2010.

${ }^{7}$ Berry, S. A., Chen, Fang-Jenq, Wilder, M. C., and Reda, D. C., "Boundary Layer Transition Experiments in Support of the Hypersonics Program", AIAA Paper 2007-4266, 2007.

${ }^{8}$ Beckwith, I. E., Creel, T. R., Chen, F.-J., and Kendall, J. M., "Free-Stream Noise and Transition Measurements on a Cone in a Mach 3.5 Pilot Low-Disturbance Tunnel," NASA TP-2180, 1983.

${ }^{9}$ Owens, L. R., Kegerise , M. A., Wilkinson, S. P. "Off-Body Boundary-Layer Measurement Techniques Development for Supersonic Low-Disturbance Flows", AIAA Paper 2011-284, 2011.

${ }^{10}$ Kegerise, M. A., Owens, L. R. and King, R. A. "High-Speed Boundary-Layer Transition Induced by an Isolated Roughness Element," AIAA Paper 2010-4999, 2010.

11 White, J. A., and Morrison, J. H., "A Pseudo-Temporal Multi-Grid Relaxation Scheme For Solving the Parabolized Navier-Stokes Equations,” AIAA Paper No. 99-3360, June 1999.

${ }^{12}$ Malik, M. R., "eMalik3d: An e $\mathrm{N}^{\mathrm{N}}$ Code for Three-Dimensional Flow over Finite-Swept Wings", High Technology Report No. HTC-9502, 1995.

${ }^{13}$ Dagenhart, J. R. and Saric, W. S., "Crossflow Stability and Transition Experiments in Swept-Wing Flow", NASA

TP 1999-209344, 1999. 
Table 1. Pressure Port Coordinates, all dimensions in $\mathbf{m m}$ except $\Theta$ is in degrees from vertical as shown in Fig. 2.

\begin{tabular}{|c|c|c|c|}
\hline \multicolumn{4}{|c|}{ Centerline Pressure Ports } \\
\hline Sx & $\mathbf{X}$ & $\mathrm{Y}$ & $\mathbf{Z}$ \\
\hline 46.761 & 46.505 & $+/-4.888$ & 0 \\
\hline 59.461 & 59.136 & $+/-6.215$ & 0 \\
\hline 72.161 & 71.766 & $+/-7.543$ & 0 \\
\hline 84.861 & 84.397 & $+/-8.87$ & 0 \\
\hline 97.561 & 97.027 & $+/-10.198$ & 0 \\
\hline 110.261 & 109.657 & $+/-11.525$ & 0 \\
\hline 122.961 & 122.288 & $+/-12.853$ & 0 \\
\hline 135.661 & 134.918 & $+/-14.18$ & 0 \\
\hline 148.361 & 147.549 & $+/-15.508$ & 0 \\
\hline 161.061 & 160.179 & $+/-16.836$ & 0 \\
\hline 173.761 & 172.81 & $+/-18.163$ & 0 \\
\hline 186.461 & 185.44 & $+/-19.491$ & 0 \\
\hline 199.161 & 198.07 & $+/-20.818$ & 0 \\
\hline 211.861 & 210.701 & $+/-22.146$ & 0 \\
\hline 224.561 & 223.331 & $+/-23.473$ & 0 \\
\hline
\end{tabular}

\begin{tabular}{|c|c|c|c|c|}
\hline \multicolumn{5}{|c|}{ First Spanwise Pressure Port Row } \\
\hline Sx & $\boldsymbol{\theta}$ & $\mathbf{X}$ & $\mathrm{Y}$ & $\mathbf{Z}$ \\
\hline 72.16 & 0.0 & 71.766 & 7.543 & 0.000 \\
\hline 72.16 & 0.0 & 71.766 & 7.543 & -12.700 \\
\hline 72.16 & 0.0 & 71.766 & 7.543 & -25.400 \\
\hline 72.16 & 0.0 & 71.766 & 7.543 & -38.100 \\
\hline 72.16 & 0.0 & 71.766 & 7.543 & -50.800 \\
\hline 72.16 & 0.0 & 71.766 & 7.543 & -63.500 \\
\hline 72.16 & 0.0 & 71.766 & 7.543 & -76.200 \\
\hline 72.16 & 22.5 & 71.826 & 6.975 & -79.199 \\
\hline 72.16 & 45.0 & 71.996 & 5.351 & -81.645 \\
\hline 72.16 & 67.5 & 72.253 & 2.906 & -83.288 \\
\hline 72.16 & 90.0 & 72.559 & 0.000 & -83.871 \\
\hline 72.16 & 112.5 & 72.867 & -2.931 & -83.292 \\
\hline
\end{tabular}

\begin{tabular}{|c|c|c|c|c|}
\hline \multicolumn{5}{|c|}{ Second Spanwise Pressure Port Row } \\
\hline Sx & $\boldsymbol{\theta}$ & $\mathbf{X}$ & $\mathbf{Y}$ & $\mathbf{Z}$ \\
\hline 135.66 & 0.0 & 134.918 & 14.180 & 0.000 \\
\hline 135.66 & 0.0 & 134.918 & 14.180 & -12.700 \\
\hline 135.66 & 0.0 & 134.918 & 14.180 & -25.400 \\
\hline 135.66 & 0.0 & 134.918 & 14.180 & -38.100 \\
\hline 135.66 & 0.0 & 134.918 & 14.180 & -50.800 \\
\hline 135.66 & 0.0 & 134.918 & 14.180 & -70.587 \\
\hline 135.66 & 22.5 & 135.031 & 13.112 & -76.100 \\
\hline 135.66 & 45.0 & 135.351 & 10.059 & -80.700 \\
\hline 135.66 & 67.5 & 135.834 & 5.463 & -83.787 \\
\hline 135.66 & 90.0 & 136.409 & 0.000 & -84.883 \\
\hline 135.66 & 112.5 & 136.988 & -5.510 & -83.796 \\
\hline
\end{tabular}

\begin{tabular}{|c|c|c|c|c|}
\hline \multicolumn{5}{|c|}{ Third Spanwise Pressure Port Row } \\
\hline Sx & $\boldsymbol{\theta}$ & $\mathbf{X}$ & $\mathbf{Y}$ & $\mathbf{Z}$ \\
\hline 199.16 & 0.0 & 198.070 & 20.818 & 0.000 \\
\hline 199.16 & 0.0 & 198.070 & 20.818 & -12.700 \\
\hline 199.16 & 0.0 & 198.070 & 20.818 & -25.400 \\
\hline 199.16 & 0.0 & 198.070 & 20.818 & -38.100 \\
\hline 199.16 & 0.0 & 198.070 & 20.818 & -50.800 \\
\hline 199.16 & 0.0 & 198.070 & 20.818 & -64.999 \\
\hline 199.16 & 22.5 & 198.235 & 19.249 & -73.001 \\
\hline 199.16 & 45.0 & 198.706 & 14.768 & -79.754 \\
\hline 199.16 & 67.5 & 199.415 & 8.021 & -84.287 \\
\hline 199.16 & 90.0 & 200.258 & 0.000 & -85.895 \\
\hline 199.16 & 112.5 & 201.109 & -8.089 & -84.300 \\
\hline
\end{tabular}

Table 2. Thermocouple locations, all dimensions in $\mathbf{m m}$.

\begin{tabular}{|c|c|c|}
\hline \multicolumn{3}{|c|}{ Leading Edge Thermocouples } \\
\hline $\mathbf{X}$ & $\mathbf{Y}$ & $\mathbf{Z}$ \\
\hline 25.4 & 0 & -41.275 \\
\hline 25.4 & 0 & 6.35 \\
\hline 25.4 & 0 & 41.275 \\
\hline
\end{tabular}

\begin{tabular}{|c|c|c|}
\hline \multicolumn{3}{|c|}{ Thermocouples in Conical Side } \\
\hline $\mathbf{X}$ & $\mathbf{Y}$ & $\mathbf{Z}$ \\
\hline 72.56 & 0 & 76.2 \\
\hline 200.26 & 0 & 76.2 \\
\hline
\end{tabular}

\begin{tabular}{|c|c|c|c|}
\hline \multicolumn{4}{|c|}{ Thermocouples Under Test Regions } \\
\hline Sx & $\mathbf{X}$ & $\mathbf{Y}$ & $\mathbf{Z}$ \\
\hline 103.91 & 103.34 & $+/-10.86$ & -41.28 \\
\hline 167.41 & 166.49 & $+/-17.5$ & -41.28 \\
\hline 103.91 & 103.34 & $+/-10.86$ & 41.28 \\
\hline 167.41 & 166.49 & $+/-17.5$ & 41.28 \\
\hline
\end{tabular}

American Institute of Aeronautics and Astronautics 


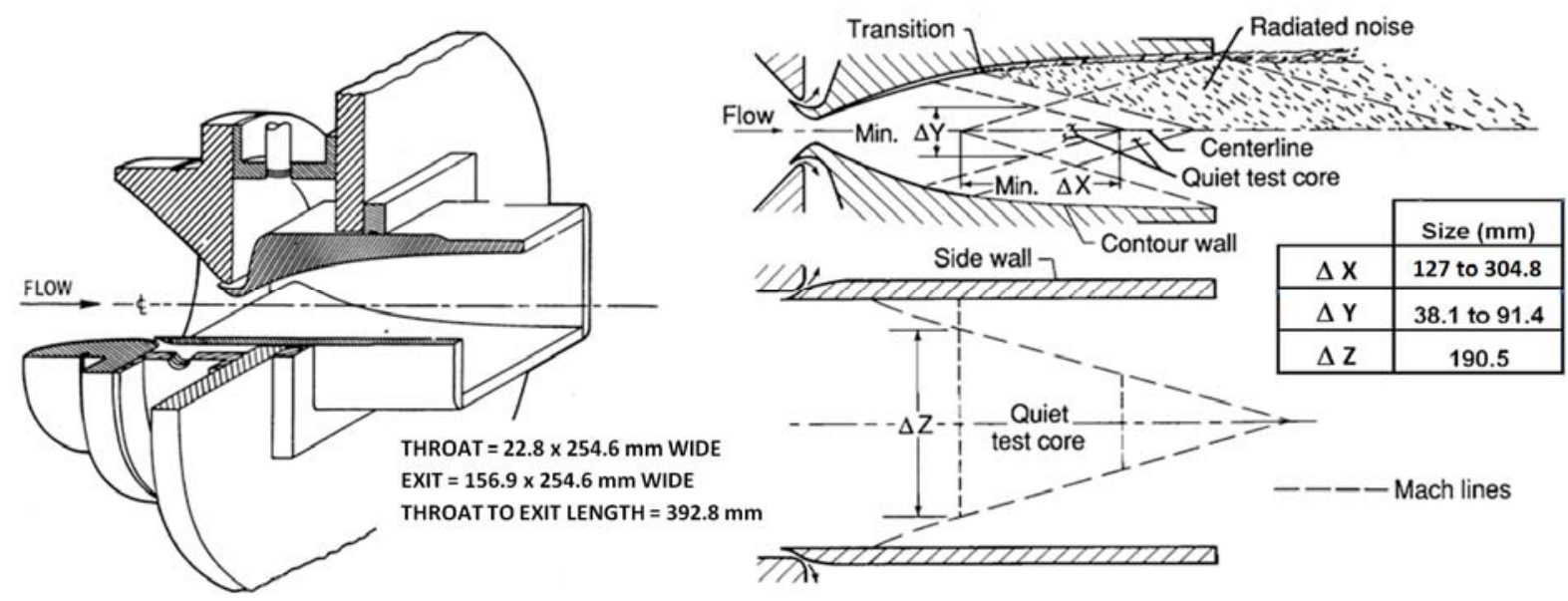

Figure 1. NASA Langley Supersonic Low Disturbance Tunnel rapid expansion rectangular nozzle.
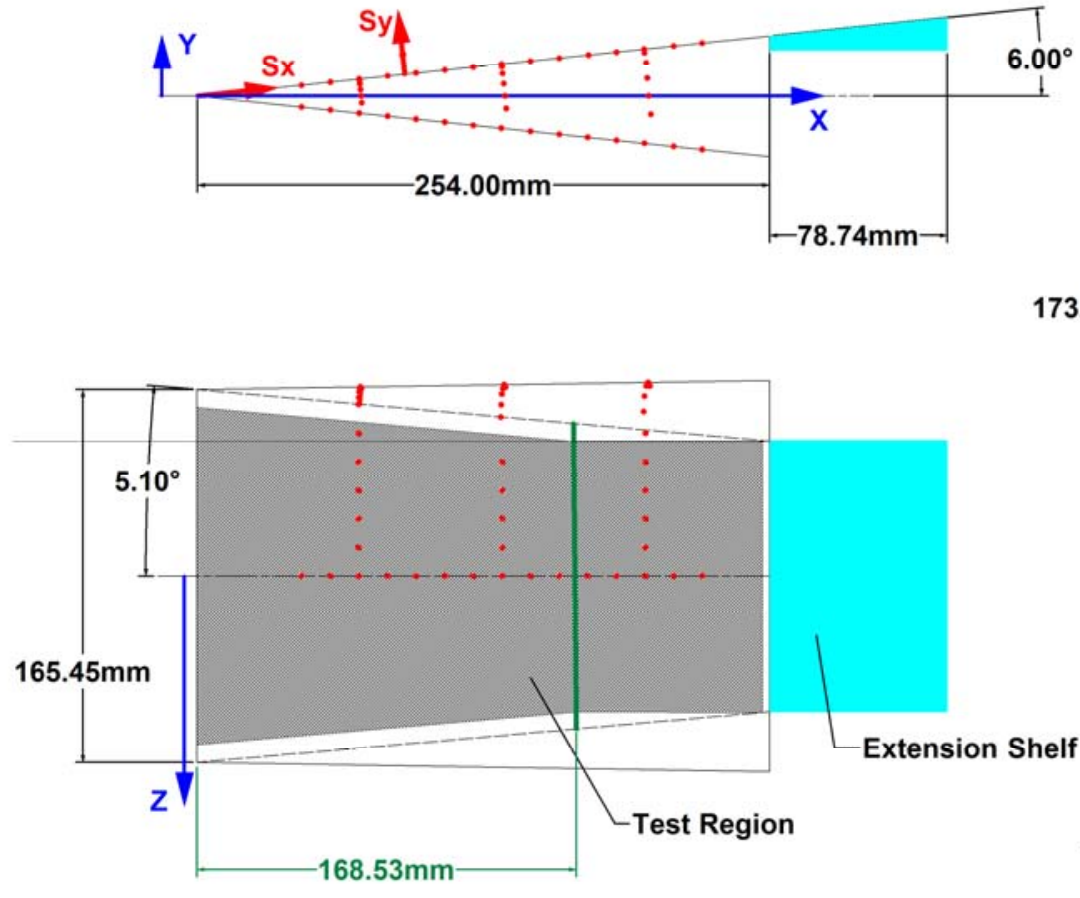

$173.88 \mathrm{~mm}$

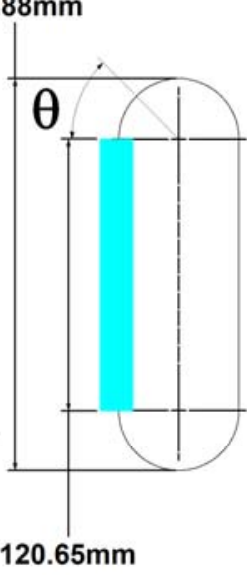

Figure 2. Wedge cone model basic dimensions, coordinate systems, extension shelf, test region, and static pressure taps shown in red. When tested without the extension shelf, the scan region is reduced to the area forward of $S x=168.53 \mathrm{~mm}(6.635 \mathrm{in}$.). Dimensions are to sharp corners. 


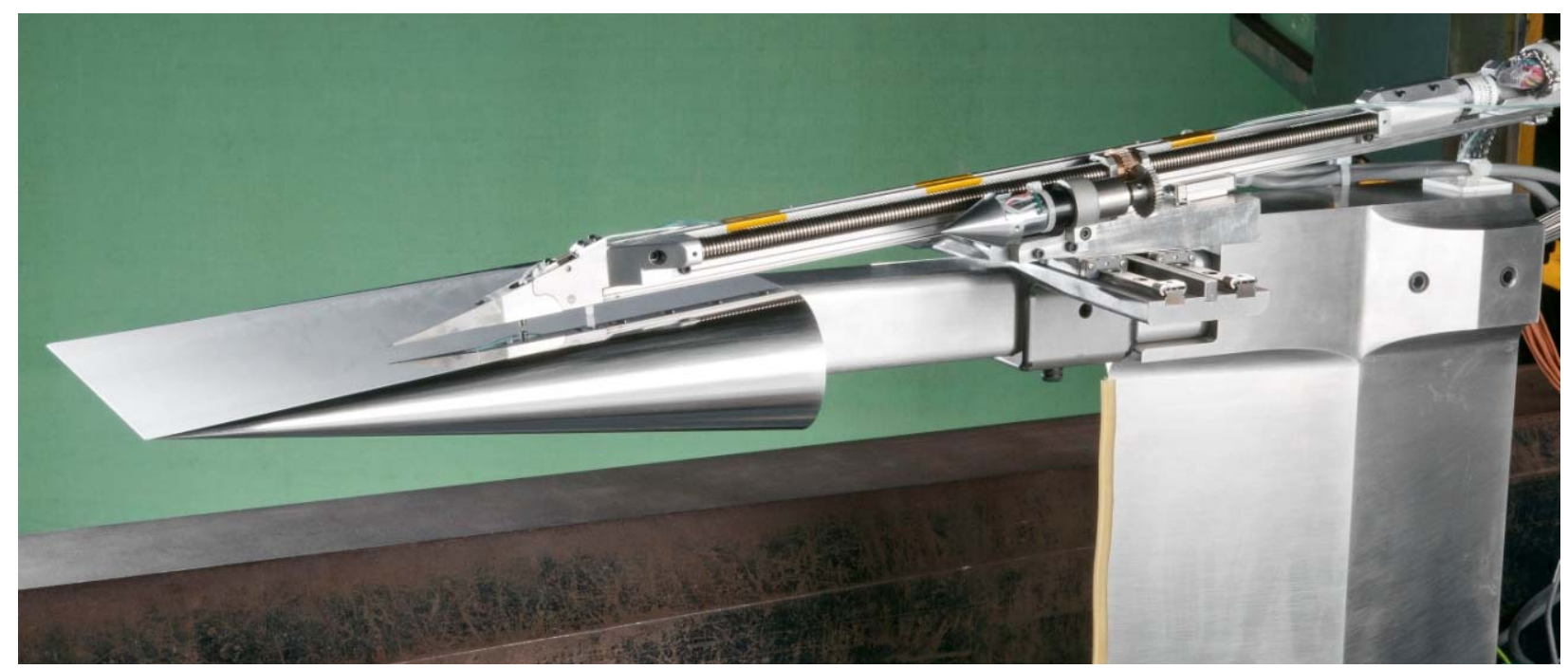

Figure 3. Picture of wedge-cone model with 3 axis traverse model as shown from first tunnel entry.

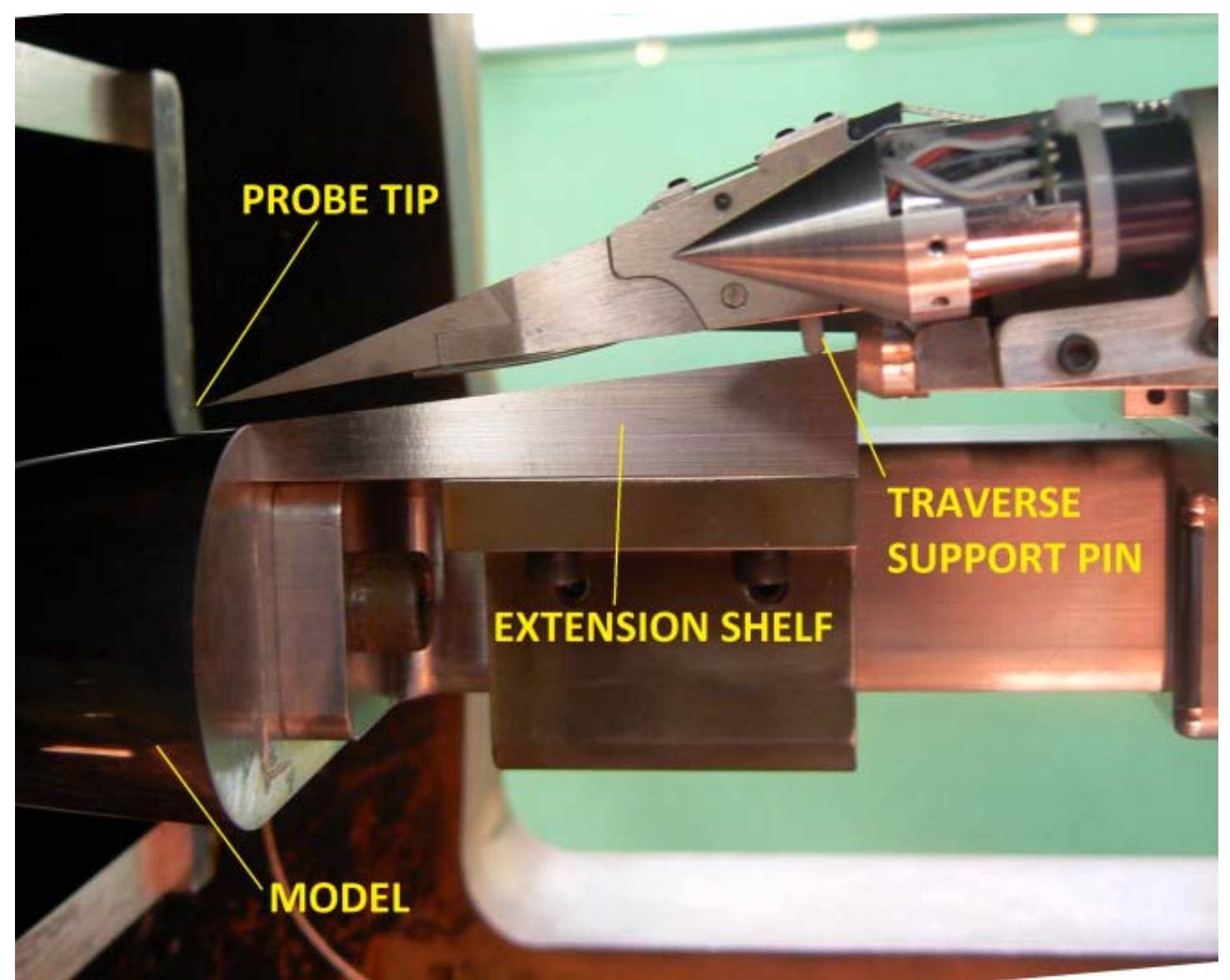

Figure 4. Typical view of traverse and model in the SLDT rapid expansion nozzle with extension shelf. Traverse shown near full aft. 


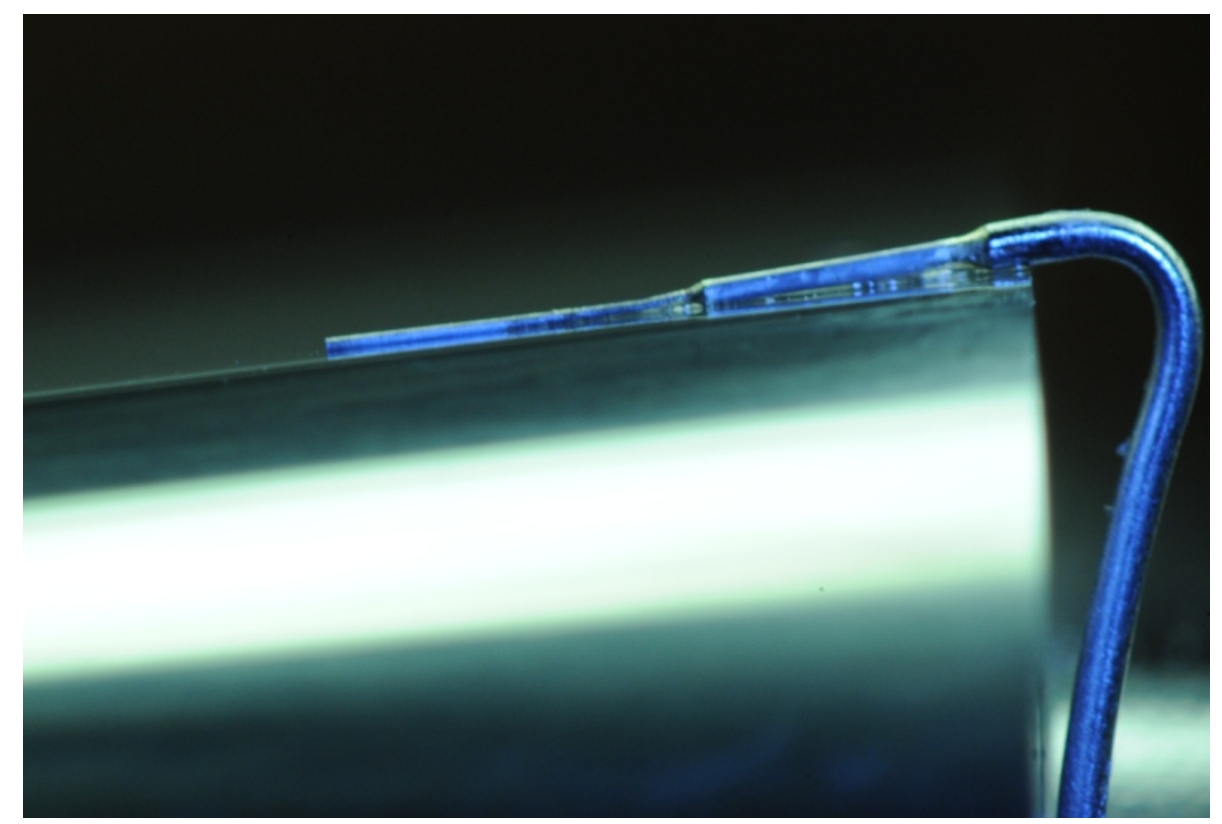

Figure 5. Preston tube at $\mathrm{Sx}=243 \mathrm{~mm}(9.57 \mathrm{in}$.$) and \mathrm{Z}=57.8 \mathrm{~mm}(2.275 \mathrm{in}$.$) .$

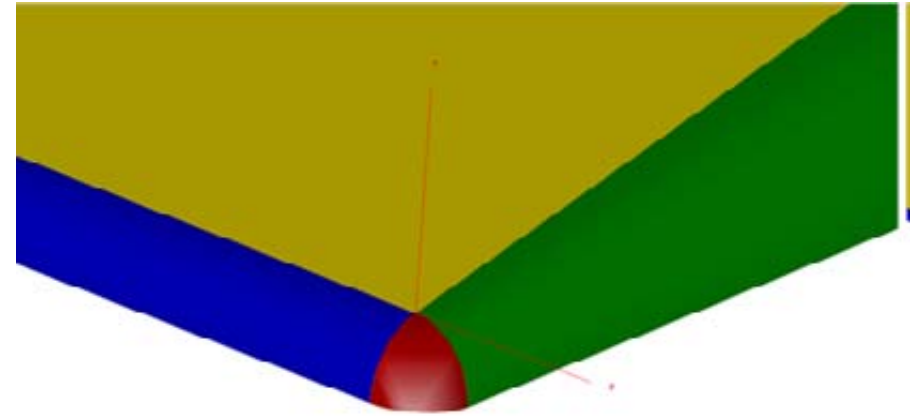

Configuration A

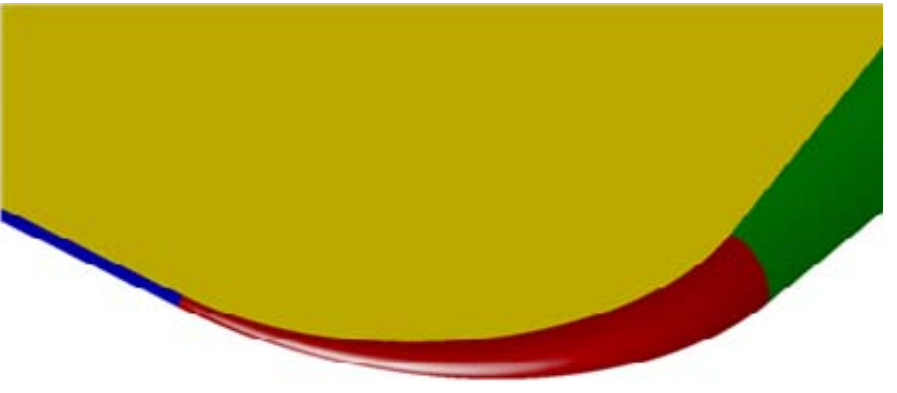

Configuration B

Figure 6. Model leading edge to cone transitions: Configuration A is a $50 \mu \mathrm{m}$ ( $0.002 \mathrm{in}$.) radius spherical transition and Configuration $B$ is a $2.54 \mathrm{~mm}(0.1 \mathrm{in}$.) planform radius transition from the $50 \mu \mathrm{m}$ radius leading edge. 


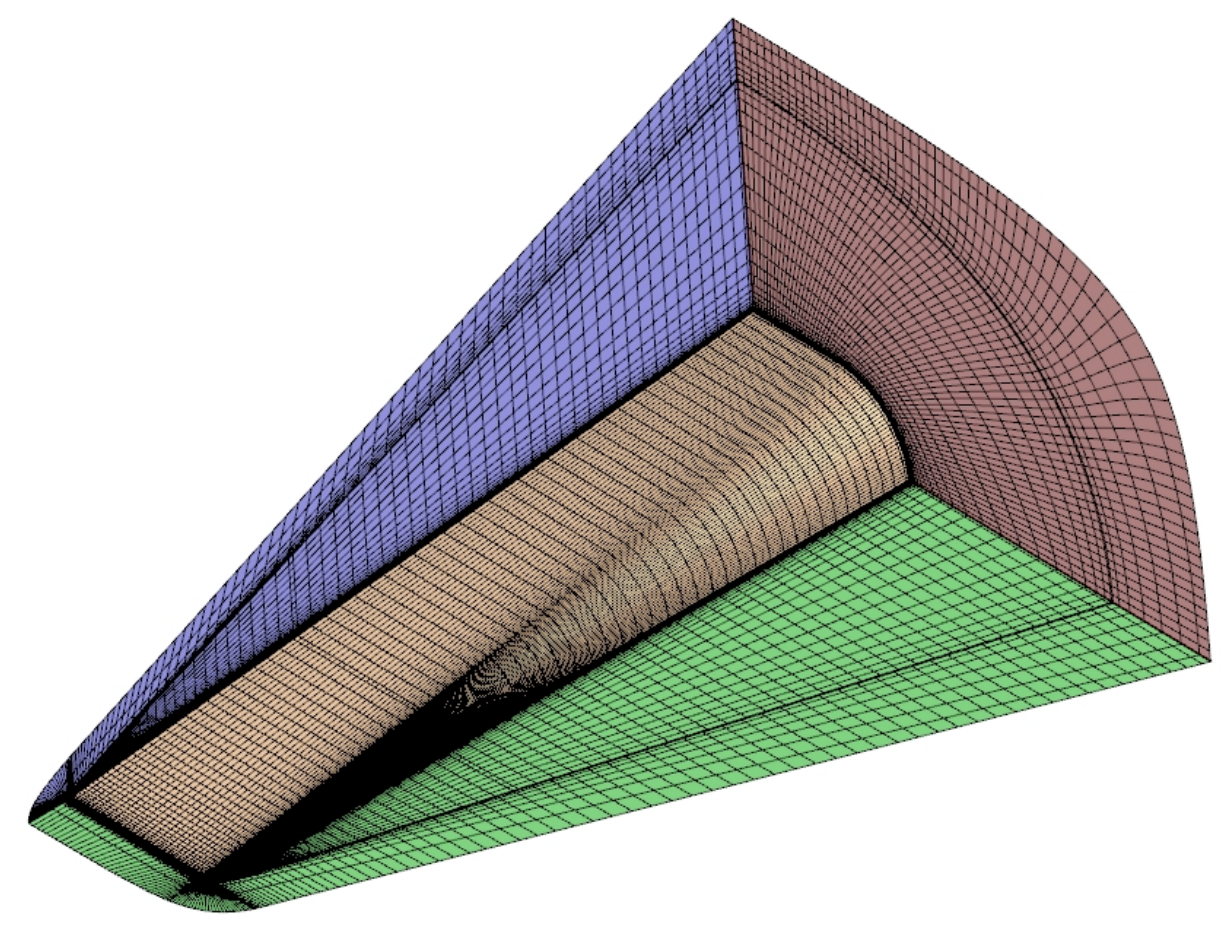

Figure 7. Meanflow computation quarter model grid with every third grid line shown.

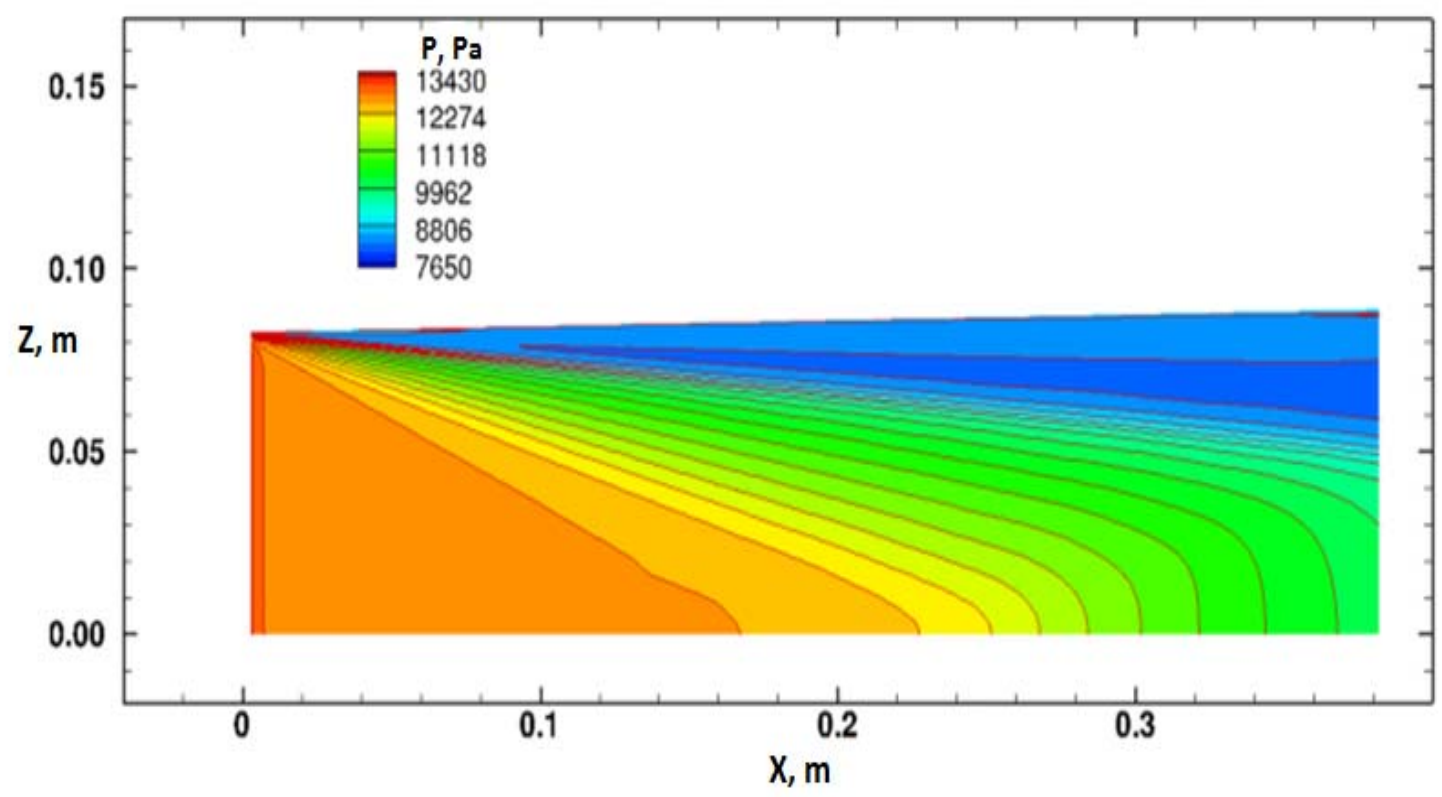

Figure 8. Computed surface pressures from VULCAN solution at $586 \mathrm{kPa}$ at zero angle of attack for configuration B corner. 


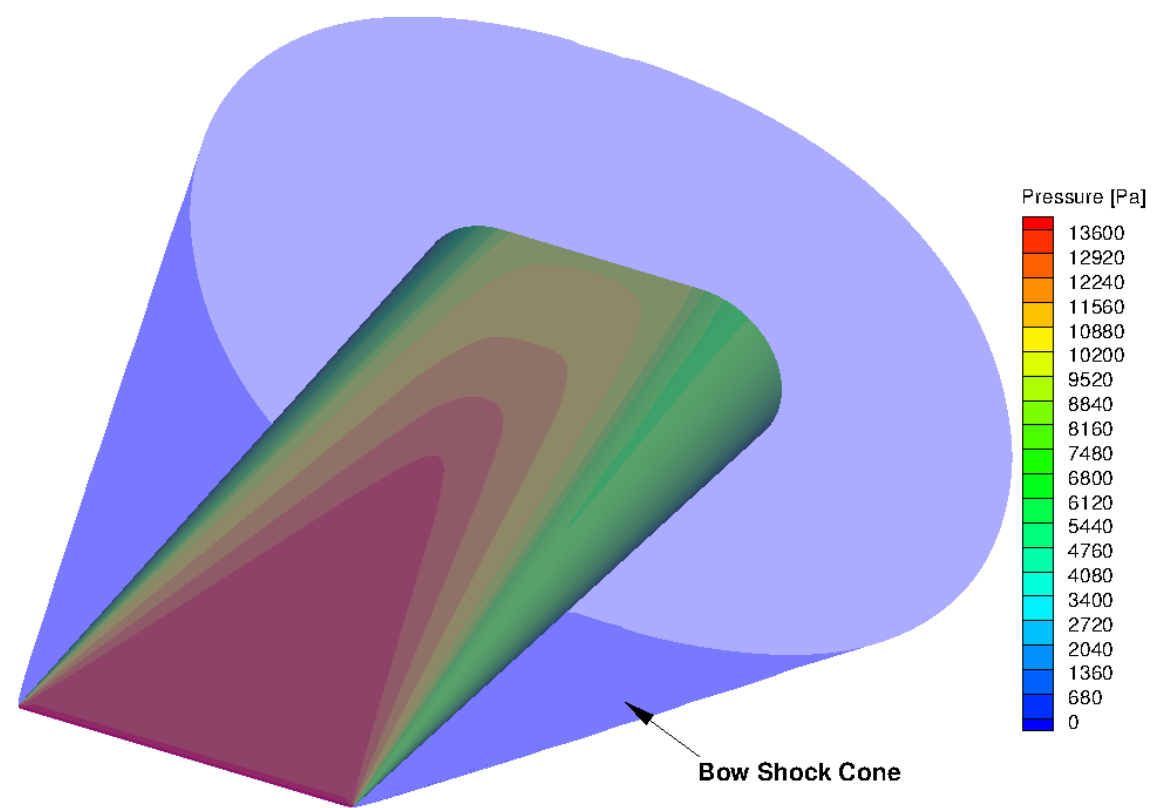

Figure 9. Isometric view of VULCAN solution showing shock capture at $586 \mathrm{kPa}$.

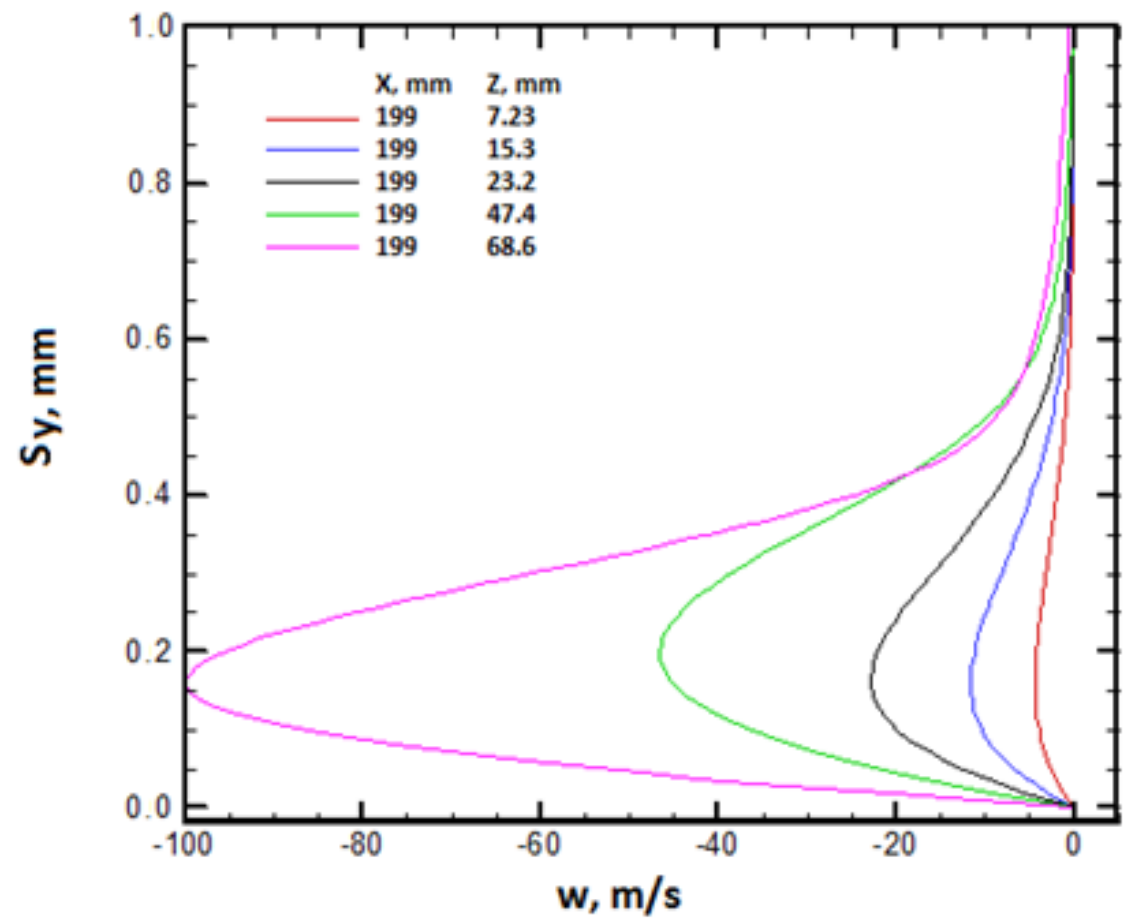

Figure 10. Computed crossflow velocity profiles at $586 \mathrm{kPa}$ from VULCAN solution. 


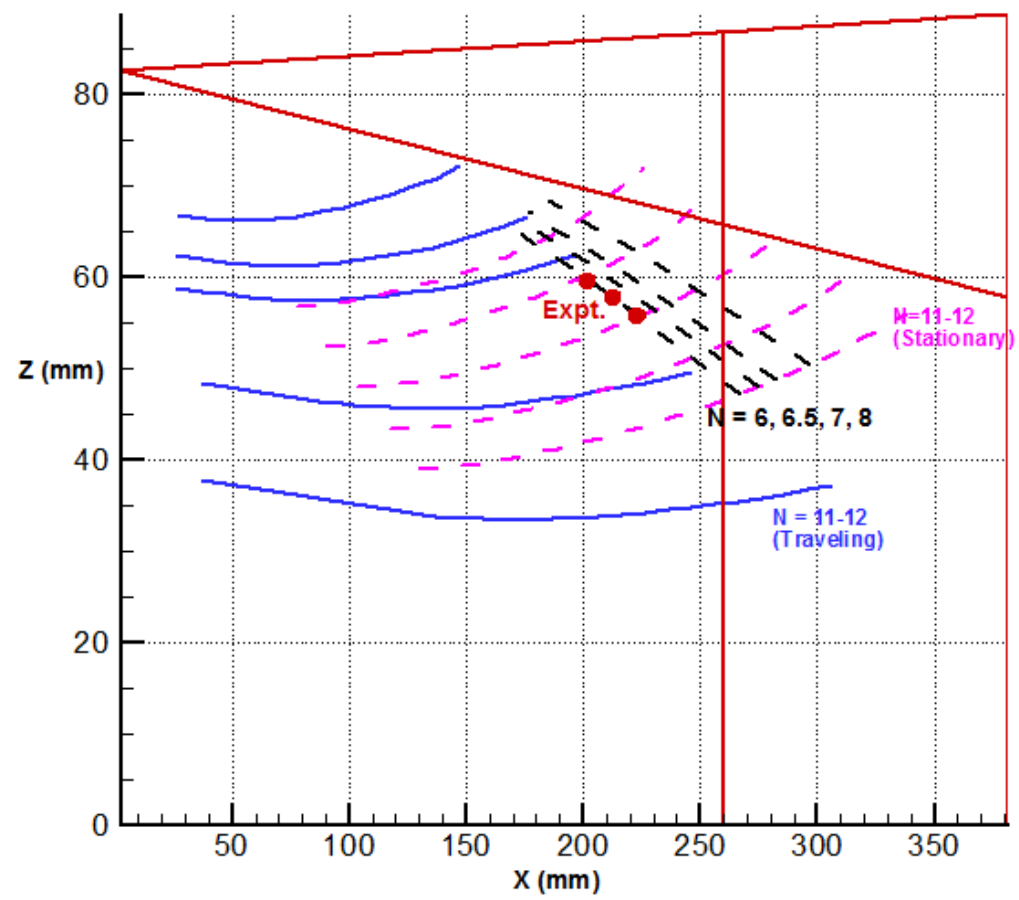

Figure 11. Transition fronts for both stationary and traveling modes at $586 \mathrm{kPa}$. The vertical red line indicates the end of the model. Points where the traverse Pitot tube was used like a Preston tube are points marked Expt.

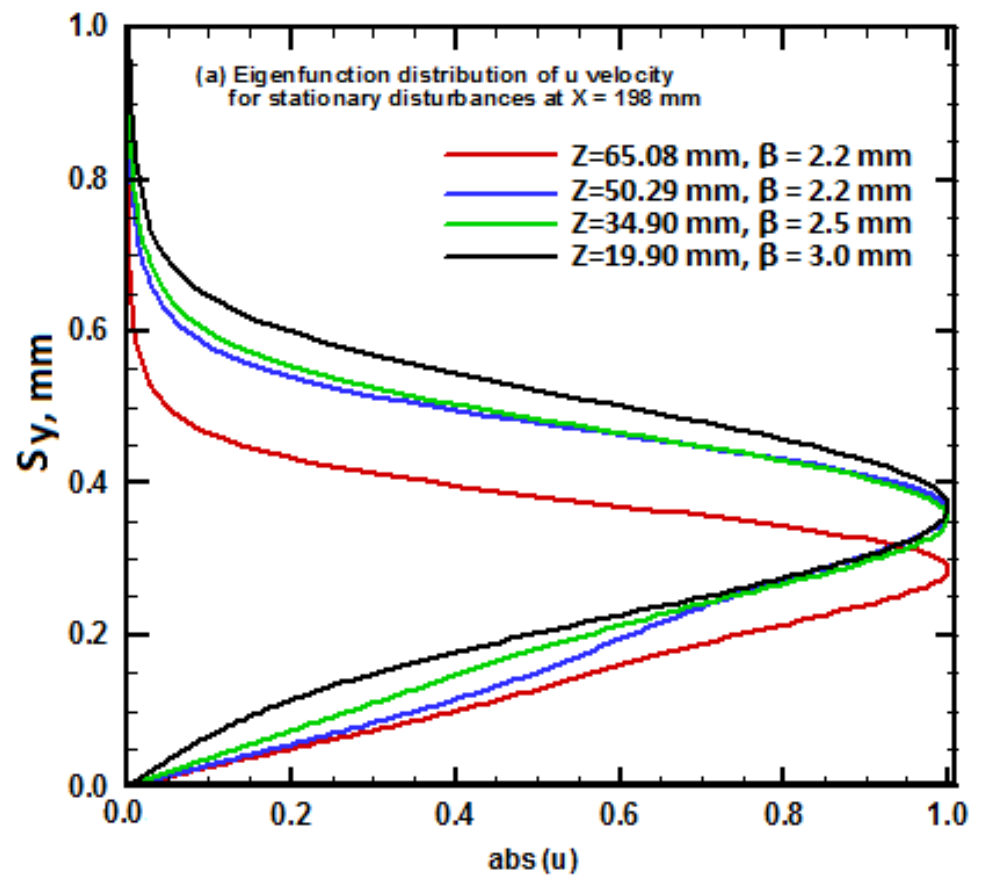

Figure 12. Spanwise eigenfunction distribution of u velocity at $X=198 \mathrm{~mm}$, stationary mode. 


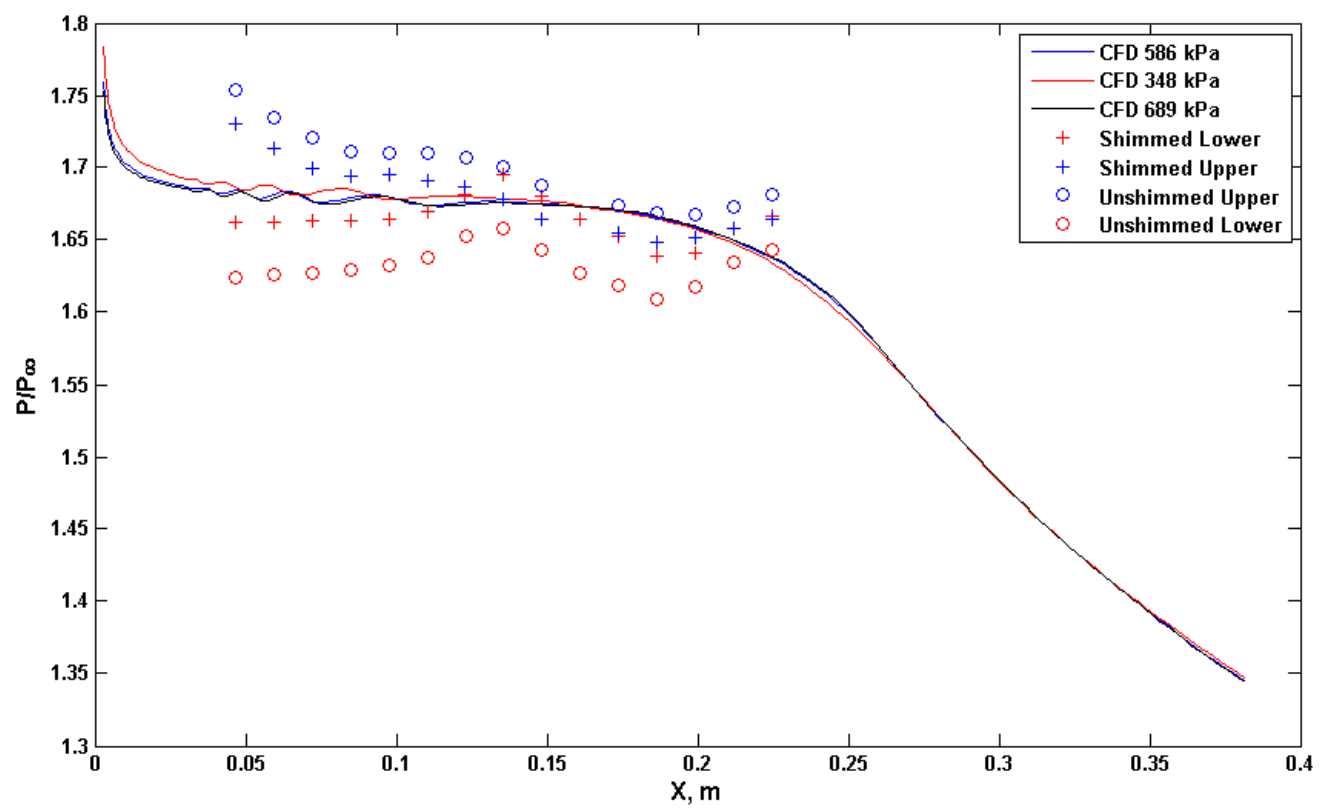

Figure 13. Centerline pressure distribution comparison between VULCAN solution and measured data at $172.4 \mathrm{kPa}$ before and after angle of attack adjustment by shim.

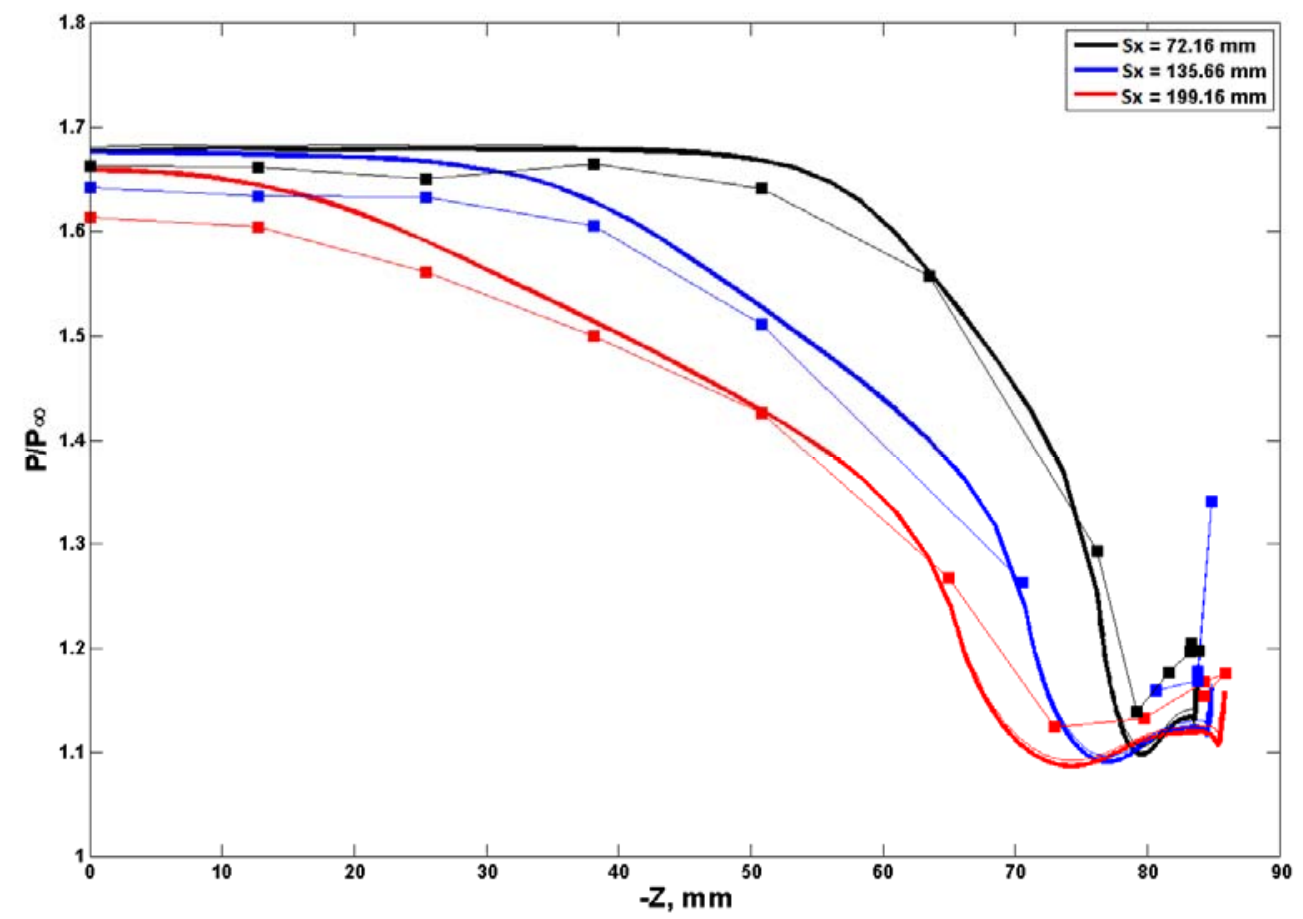

Figure 14. Spanwise pressure distribution comparison between VULCAN solution and measured data at $172.4 \mathrm{kPa}$. Heavy lines are CFD from all three pressures. Solid symbols are from the shim adjusted model. 


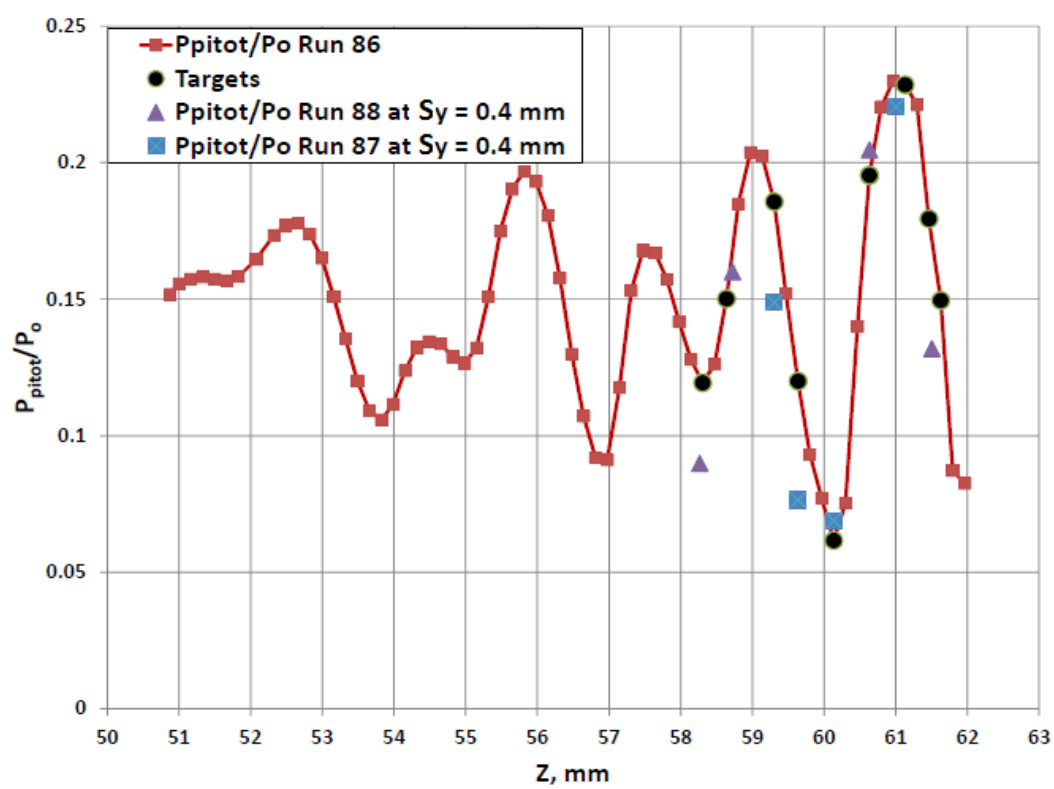

Figure 15. Spanwise survey at $\mathrm{Sx}=168.53 \mathrm{~mm}(6.635 \mathrm{in}$.) and $\mathrm{Sy}=0.4 \mathrm{~mm}$ (run 86$)$ with points from wall normal boundary layer profiles at $\mathrm{Sy}=0.4 \mathrm{~mm}$ from first entry.

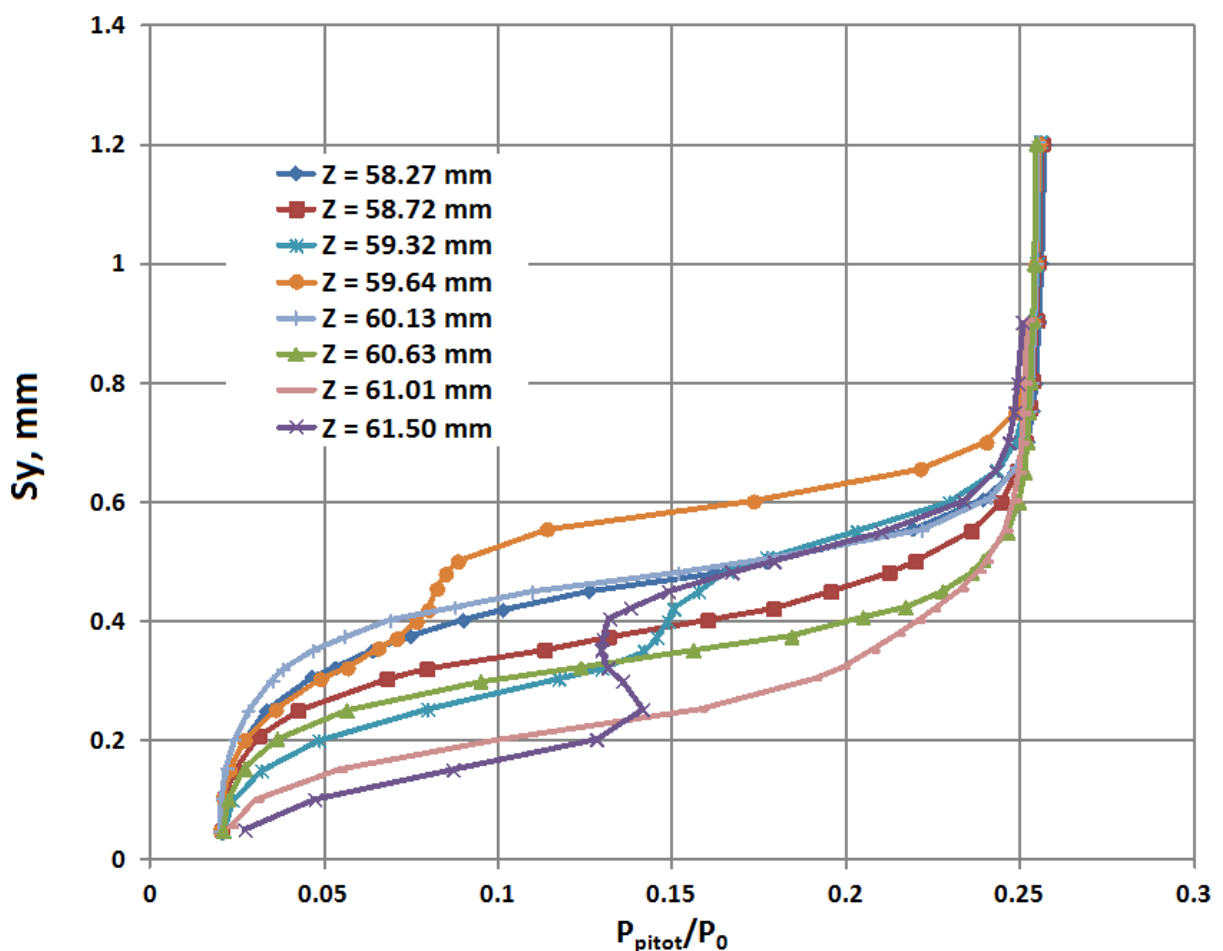

Figure 16. Boundary layer profiles at $\mathrm{Sx}=168.53 \mathrm{~mm}(6.635 \mathrm{in}$.) providing the boundary layer points of Fig. 15 and the contour plot data showing the crossflow vortical structure of Fig. 17. 


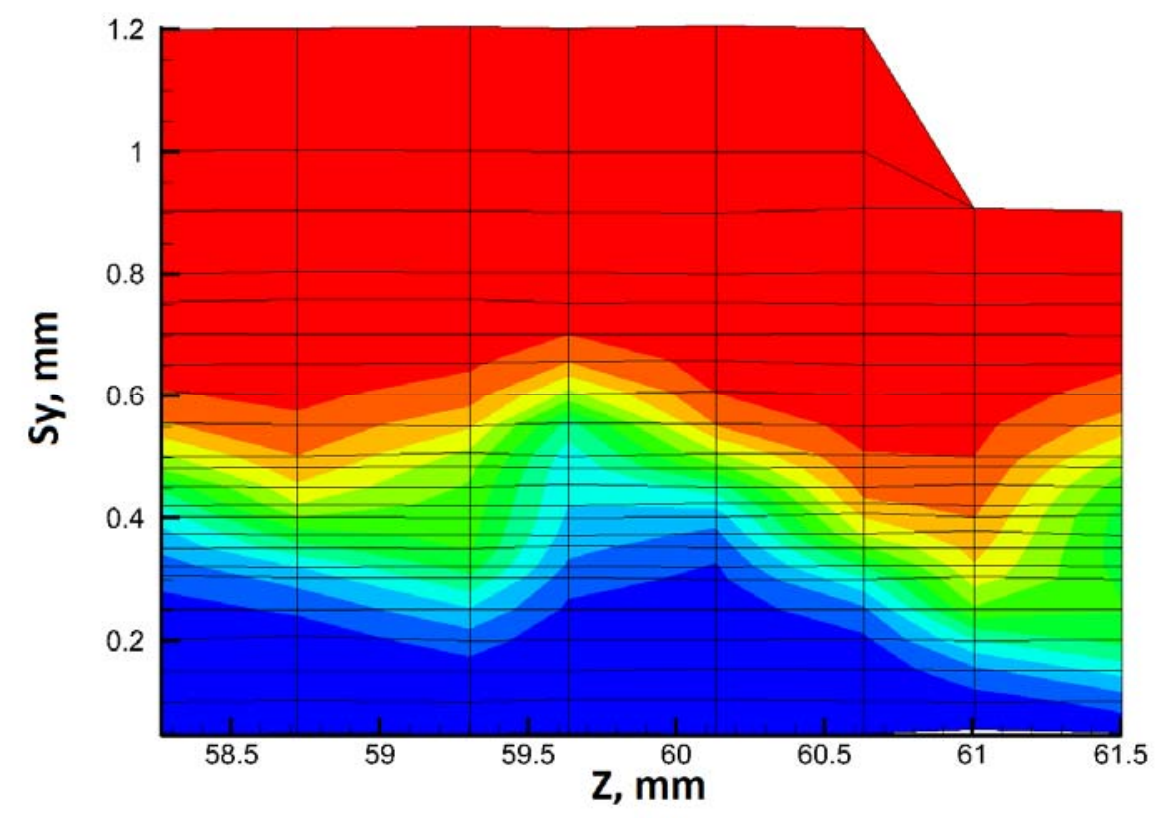

Figure 17. Boundary layer profile data from Fig. 16 showing resolution of vortices.

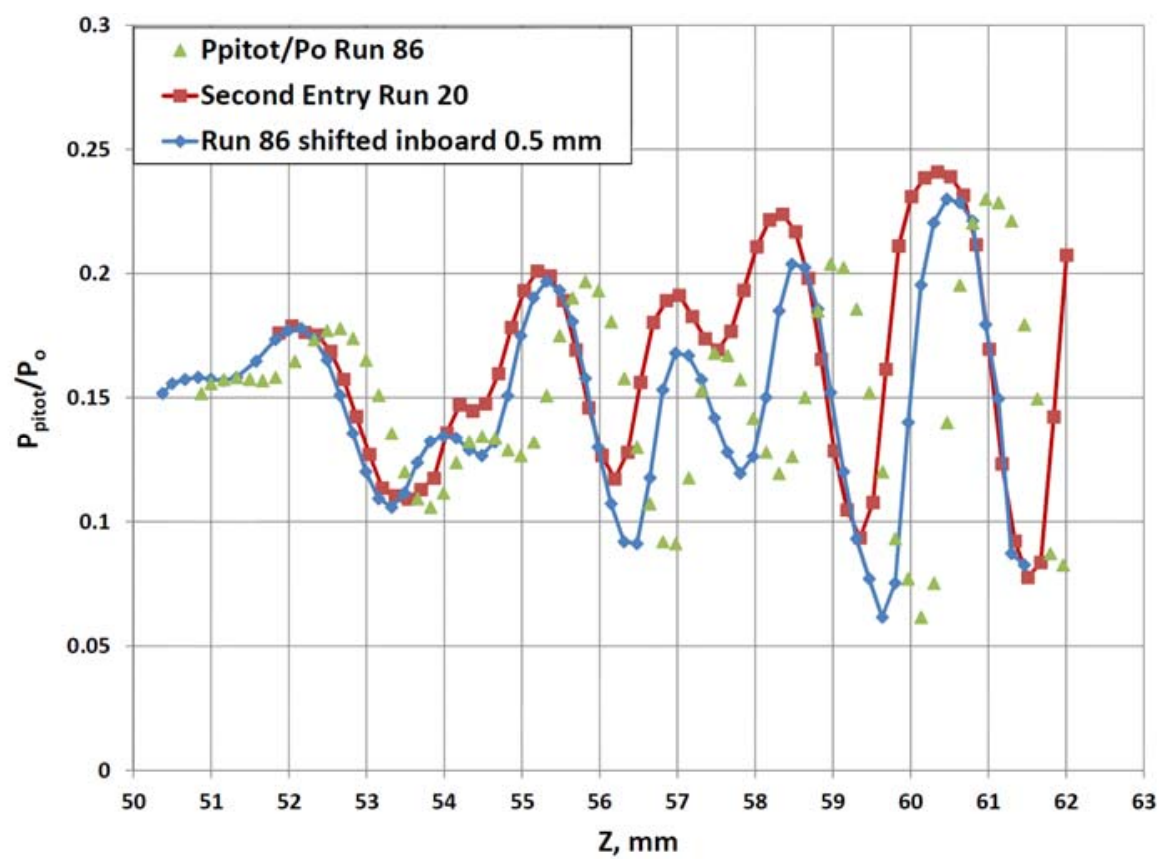

Figure 18. Lateral scans from first and second test entries at $S x=168.53 \mathrm{~mm}(6.635 \mathrm{in}$.$) at S y=0.4 \mathrm{~mm}$ showing repeatability with shift explained in paper. 


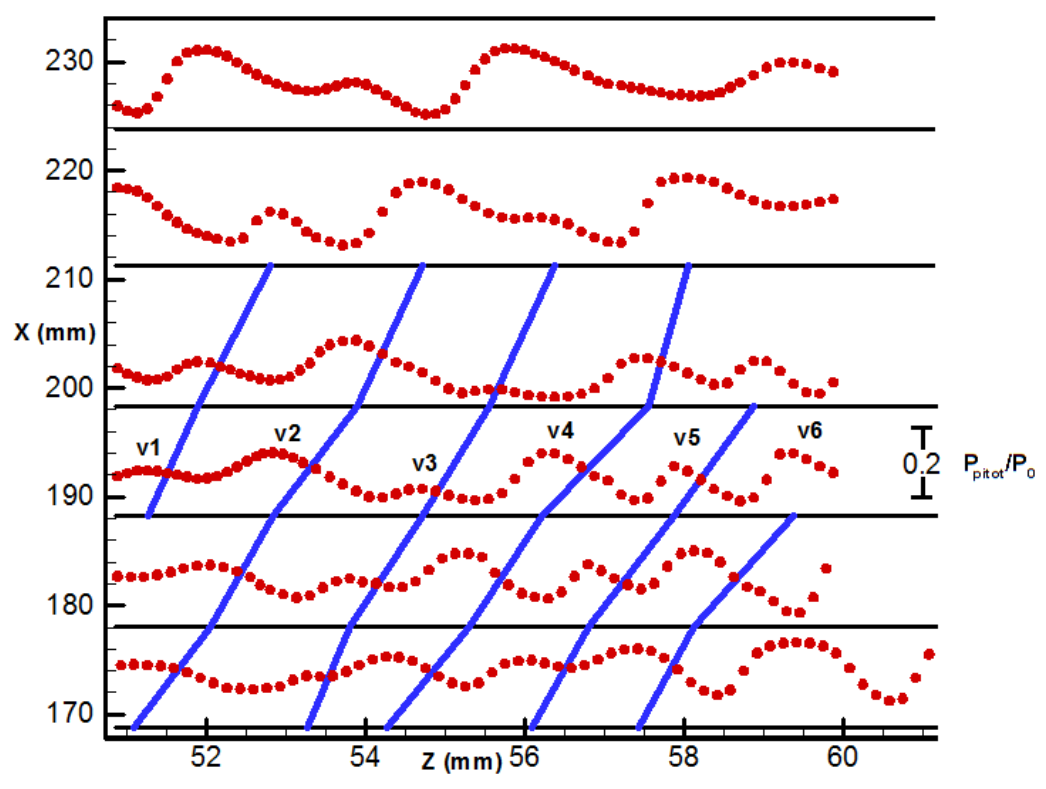

Figure 19. Lateral scans at $S y=0.4 \mathrm{~mm}$ (symbols only) with traces of peak $P_{\text {pitot }} / P_{0}$ at $586 \mathrm{kPa}$. Representative line indicating pressure scale shown on right.

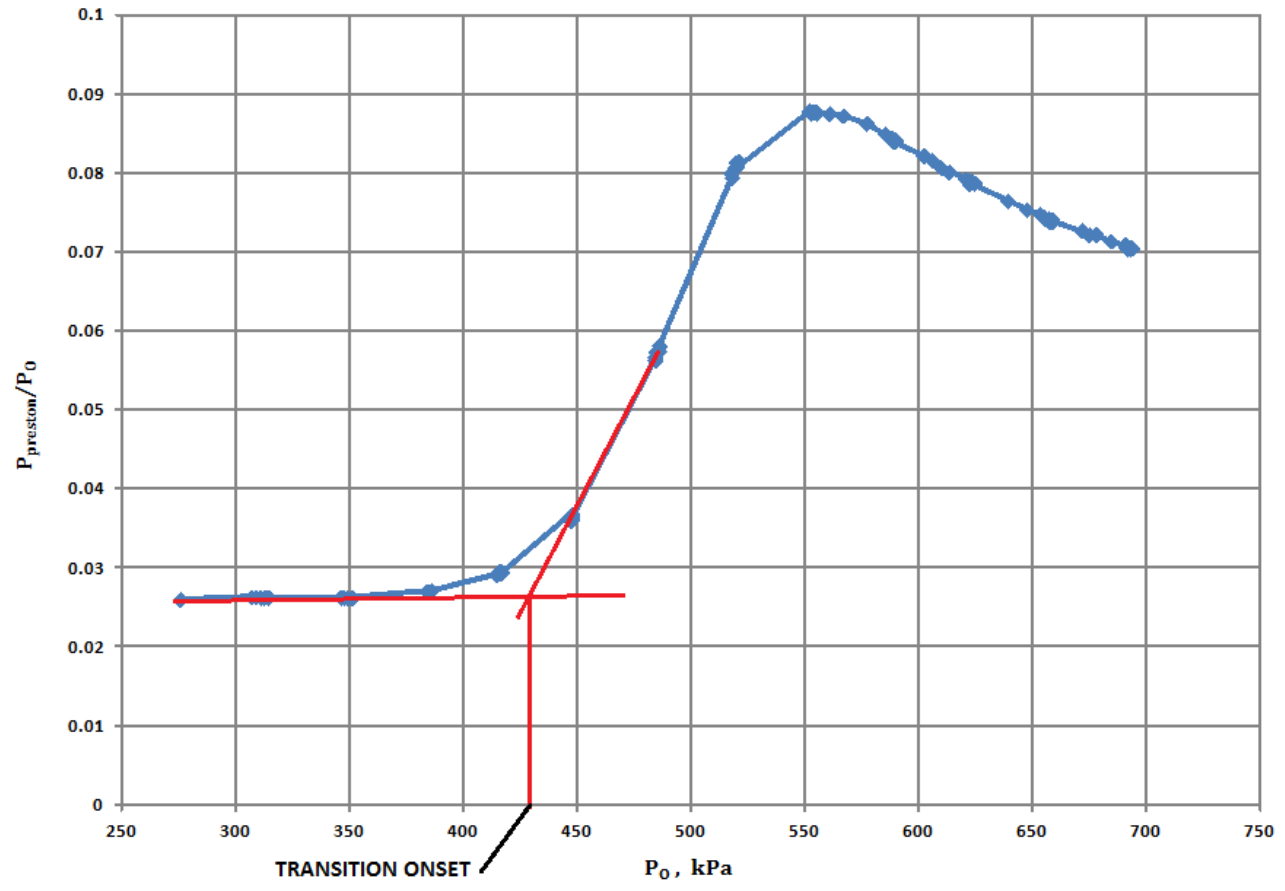

Figure 20. Preston tube measurement indicating transition on the crossflow model. Red lines indicate method used for finding transition onset pressure. 


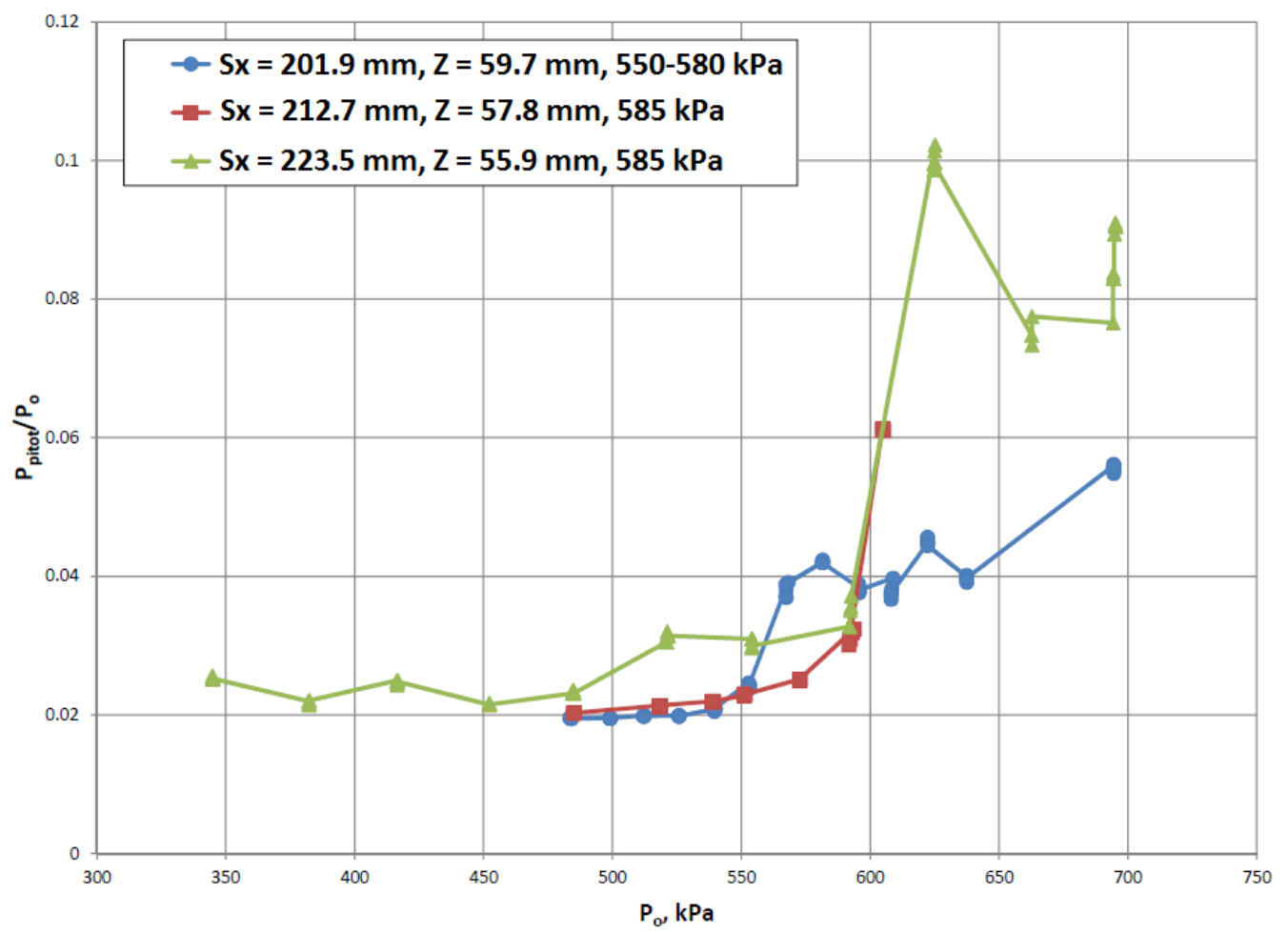

Figure 21. Fouled to surface Pitot pressure data with approximate transition onset pressures noted. 